\title{
Mean crossover functions for uniaxial 3D Ising-like systems
}

\author{
Yves Garrabos \\ Equipe du Supercritique pour l'Environnement, les Matériaux et l'Espace, \\ Institut de Chimie de la Matière Condensée de Bordeaux - UPR 9048, \\ Centre National de la Recherche Scientifique - Université Bordeaux I - 87, \\ avenue du Docteur Schweitzer, F 33608 PESSAC Cedex, France \\ Claude Bervillier \\ Laboratoire de Mathématiques et Physique Théorique, CNRS UMR 6083, \\ Université de Tours, Parc de Gandmont, 3 r200 Tours, France.
}

(Dated: 05 April 2006)

\begin{abstract}
We give simple expressions for the mean of the max and min bounds of the critical-to-classical crossover functions previously calculated [Bagnuls and Bervillier, Phys. Rev. E 65, 066132 (2002)] within the massive renormalization scheme of the $\Phi_{d}^{4}(n)$ model in three dimensions $(d=3)$ and scalar order parameter $(n=1)$ of the Ising-like universality class. Our main motivation is to get efficient theoretical expressions to coherently account for many measurements performed in systems where the approach to the critical point is limited but yield data which are still reproducible by the $\Phi_{d}^{4}(n)$ model (like in the subclass of one-component fluids).

PACS numbers: 64.60.Ak., 05.10.Cc., 05.70.Jk, 65.20.+w
\end{abstract}

\section{INTRODUCTION}

The universal features of the three-dimensional (3D) Ising-like systems close to their critical points are now well-established by the renormalization group (RG) approach [1]. In this theoretical context the Ising-like universality is attached to the existence of a unique nontrivial fixed point (the Wilson-Fisher fixed point [2] noted W-FP in the following) which any Hamiltonian representation of an actual system at criticality is driven to under the action of the renormalization transformations [3].

Less known is the existence of theoretical expressions, obtained using perturbative field theory (FT) techniques $[4,[5,6,67,8]$, which are used to interpolate between the critical (non-classical) behavior (controlled by the WFP) and a classical behavior (controlled by the Gaussian fixed point, noted G-FP in the following). Such theoretical expressions are customarily named classical-to-critical crossover functions.

Actual systems undergoing a second order phase transition also display a kind of "classical"-to-critical crossover but it is not of the same nature as the theoretical one alluded to above. In actual systems, the "classical" part is only a non-critical part (not governed by the G-FP) whereas in the theoretical interpolation, the classical part still belongs to a critical domain (governed by the G-FP). Moreover, the renormalization procedure of FT is reductive in the sense that many sources of non-universality are discarded because they are unessential in the vicinity of the critical point. However, non-universal features become more and more important as one moves away from the critical point. Finally, these non-universal characteristics are responsible for the distinction between the nonasymptotic critical behavior and the asymptotic critical behavior.

Generally, one cannot expect to observe an agreement between the theoretical crossover functions and the experimental data in a wide domain ranging from the close vicinity of the critical point down to a state far away from it. Nevertheless, exceptions may exist as in the case of the "subclass" of one-component fluids.

It has been shown $9,10,11,12,13$ ] that, despite their wide range of numerical values, the one component fluid data collected in the literature regarding a given property (susceptibility, order parameter density, correlation length, etc.) can be reduced to a unique scaling curve (also called master curve) over a wide critical domain. Now, it is well known that this scaling curve can be well reproduced by the classical-to-critical crossover functions of FT [13. However, an accurate understanding of the range of its validity is lacking. In the present paper, we provide modified versions (named "mean crossover functions") of the theoretical forms given in references 5, 6, 14]. A careful attention is given to the characterization of the Ising like preasymtotic domain where the number and the nature of adjustable parameters can be easily controlled. A forthcoming paper [15] will illustrate the application of the mean crossover functions to provide a precise criterion for estimating the effective extension of the critical asymptotic domain of the fluid subclass, a key point for future developments of a complete equation of state (e.o.s.) for fluids.

Actually, since it is difficult to approach accurately the critical point both experimentally and theoretically, the effective (observed) scaling domain is limited towards the critical point whereas, in the opposite direction, the scaling curve stops when the systems are no longer critical and before they display any kind of "classical behavior". Here classical behavior means the "critical" behavior controlled by the G-FP. Consequently, most of the useful data on a system near criticality belong to an intermediate (nonasymptotic) regime where it is not valid to repro- 
duce the data with the currently used expansion limited to a pure power law eventually corrected by one Wegner's term [16] (the validity of such a form implies the close vicinity of the critical point).

For development of an e.o.s., it is of great importance to have theoretical expressions which reproduce the phenomenologically observed scaling curves and which allow one to situate unambiguously any data set with respect to the actual (system dependent) field distance to the critical point. It must be stressed that, in this view, the aim is not to test the most refined theoretical hypotheses, or the most precise estimates of the universal numbers associated with the asymptotic or pre-asymptotic critical behavior. In addition, our scheme only applies to the primary critical paths along the critical isochore in the homogeneous and the non homogeneous domain, without any supplementary consideration on the roles played by other nonuniversal scales associated with the eventual effects such as non symmetry [17] and mixing [18] of the scaling field variables very close to the critical point.

$>$ From a pioneering study [19] of the available data on xenon [20, 21], it is known that the classical-to-critical crossover functions obtained from the $\Phi_{d}^{4}(n)$ model (with $d=3$ and $n=1$ ) fit the one-component fluid results, introducing explicit determination of the fluid-dependent scale factor for the relative temperature field. More recently, a similar study [22] of several measurements near the liquid-gas critical point of ${ }^{3} \mathrm{He}$ [23], has provided an equivalent conclusion, demonstrating that, ${ }^{3} \mathrm{He}$ as a simple fluid, is also situated very close to a renormalized trajectory that links the G-FP to the W-FP. However, the authors of Ref [4, 5, 6, 14] have given a practical (max or min) form of their effective functions which is better adapted to the eventual experimental test of the "best" theoretical estimates of the asymptotic universal quantities (attached to the close vicinity of the critical point), rather than to an unambiguous characterization of a nonasymptotic critical domain of a given subclass of systems. In fact, accounting for the error estimates attached to the universal critical quantities under two max and min bounded sets of functions prevents the characterization of the scaling curve clearly which, of course, is currently accessible experimentally at some non-small distance (to be determined) to the critical point but also is sometimes accompanied by a relatively poor accuracy in the measurement. Moreover, due to the decrease of the theoretical error as the distance to the critical point increases, the mean values of the bounded functions are sufficient to characterize the scaling curves.

Our aim in the present paper is to determine inbetween controlled functions that are better adapted for unambiguous determination (within a current experimental mean accuracy) of the characteristics of the scaling curves of the subclass of one-component fluids. An important point to note a posteriori is the unchanged value of the temperature scale factor determined in [19]. However the uncertainty is considerably reduced showing that it was essentially generated by the theoretical uncer- tainties of the universal values calculated in the asymptotic regime. That demonstrates the need to provide mean classical-to-critical crossover functions which, by construction, have a well defined single asymptotic limit (i.e. "Ising-like-well-defined"). We will then be able to determine significative values of the scale factors which characterizes the scaling behavior occuring in the intermediate critical domain.

The theoretical crossover functions which we are interested in have been derived from a massive renormalization (MR) scheme applied to the $\Phi_{d}^{4}(n)$ model. The MR scheme has been initially developed in references [4, 5, 6], hereafter referenced MR6, using the Borel resummation technique based on the results of the sixth-loop series 24]. They have been recently revisited in reference [14], hereafter referenced MR7, to account for an extension to the seventh-loop series [25]. We do not consider here the crossover functions determined by Dohm and coworkers [7, 8] who have used another renormalization scheme (minimal) within which the known series are shorter than in the MR scheme and have provided their crossover functions under implicit forms.

The paper is organized as follows.

In Section 2, we introduce the main characteristics of the MR scheme and of the crossover functions. Special attention is given to the non-universal nature of the adjustable parameters that are introduced in the max and min theoretical functions for fitting experimental data.

In Section 3, the mean functions are determined relying on the properties of the theoretical functions in the two limiting $3 D$ Ising-like and mean field-like descriptions, respectively close to the W-FP and G-FP. Such descriptions correspond to the pre-asymptotic domains (PAD) near each fixed point where a Wegner expansion restricted to two terms (leading and first confluent terms) is valid. The Ising-like PAD includes the correlations between parameters due to the error-bar determination of the exponents and amplitude combinations very close to the W-FP. The addition of the equivalent mean field PAD description very close to the G-FP leads to a mean crossover function with a limited number of calculated parameters for this function (three in the selected example). A well-controlled form of any mean crossover function can be obtained in a similar manner, i.e. three calculated parameters for each function. In such a situation, the theoretical crossover forms are obtained for a unique value of one parameter among the three. This parameter acts then as a relative sensor to estimate the dominant nature, either (Ising-like) critical, or (mean field-like) classical, of the calculated crossover. Using this sensor, we propose an explicit criterion to measure the extension of the Ising-like PAD along the critical isochore where a four parameter characterization of each system is well-understood in scaling nature. In section 4, we provide a conclusion.

In Appendix A, assuming knowledge of the critical temperature of the system, we illustrate the threeadjustable-parameter characterization of the comparison 
with experimental data. The emphasis is on the role of a characteristic microscopic length scale, the reminiscence of which is carried by the dimension of the "bare" $\phi^{4}$ coupling $g_{0}$ [see Eq. (1) below].

Appendix B gives some details on the derivation of the mean crossover function for the particular case of the order parameter in the heterogeneous domain, with a view to better account for large (theoretical and experimental) uncertainties in the determination of its corresponding first confluent amplitude.

\section{THE MASSIVE RENORMALIZATION SCHEME}

\subsection{The model}

The calculations of the crossover functions in the $\Phi_{d}^{4}$ model rely upon the renormalization program of perturbation FT. This scheme makes the perturbative expansion of the correlation functions free of ultra-violet divergencies. Before renormalization, the dimensionless Hamiltonian (a true hamiltonian divided by $k_{B} T$, with $k_{B}$ the Boltzman constant) of the actual system reads:

$$
\begin{aligned}
\mathcal{H}=\int d^{d} x & \left\{\frac{1}{2}\left[\left(\nabla \overrightarrow{\Phi_{0}}\right)^{2}+r_{0}\left(\overrightarrow{\Phi_{0}}\right)^{2}\right]+\frac{g_{0}}{4 !}\left[\left(\overrightarrow{\Phi_{0}}\right)^{2}\right]^{2}\right. \\
+ & \left.\vec{h} \cdot \overrightarrow{\Phi_{0}}\right\}
\end{aligned}
$$

in which $d$ is the space dimension and $\vec{h}$ and $\overrightarrow{\Phi_{0}}$ are vectors of dimension $n . \quad \vec{h}$ is the (magnetic-like) ordering field vector. $\overrightarrow{\Phi_{0}}$ is the spin-like vector. The ordering field vector $\vec{h}$ is not renormalized and so will no longer be considered explicitly in the following (except when required). The coupling field $r_{0}$ and the coupling constant $g_{0}$ are the bare (physical) parameters, which are two system-dependent quantities characterizing the critical point location of the physical system.

For the sake of simplicity, we limit ourselves in the following discussion to the scalar case $n=1$.

At this stage, it is worthwhile to state that the dimension of any quantity appearing in $\mathcal{H}$ is expressed in terms of only one inverse length unit: a wave-vector cutoff, $\Lambda$ (or the inverse of a lattice spacing for magnetic solid systems for example). Since $\mathcal{H}$ is dimensionless, a simple evaluation of Eq. (11) shows that the dimension (in unit $\Lambda$ ) of $\Phi_{0}$ is $\frac{d-2}{2}$, while that of $r_{0}$ is 2 , and that of $g_{0}$ is $4-d$.

When $d=4$, the perturbative expansion in powers of the dimensionless coupling constant $g_{0}$ involves ultraviolet divergencies which are removed by redefining the three initial Hamiltonian (bare) parameters $\left\{\Phi_{0}, r_{0}, g_{0}\right\}$ into new (renormalized) ones $\{\Phi, \bar{m}, g\}$, via the following relations:

$$
r_{0}=\bar{m}^{2}+\delta \bar{m}^{2}
$$

$$
\begin{gathered}
\Phi_{0}=\left[Z_{3}(g)\right]^{\frac{1}{2}} \Phi \\
g_{0}=(\bar{m})^{4-d} g \frac{Z_{1}(g)}{\left[Z_{3}(g)\right]^{2}}
\end{gathered}
$$

with subtraction conditions which, in four dimensions $(d=4)$, are required to make the renormalized perturbative expansion in powers of $g$ finite.

In three dimensions, however, only the mass renormalization is needed to have a finite theory. Consequently, with the introduction of $\bar{m}$ instead of $r_{0}$, the renormalization functions, like $Z_{1}$ and $Z_{3}$, even expressed in terms of the "bare" coupling $g_{0}$ and without ultra-violet regulator $\Lambda$ (set to infinity), are well defined functions. Introducing the notation $\Gamma_{0}^{(L, N)}\left(\{q, p\} ; \bar{m}, g_{0}, d\right)$ for the Fourier transforms of the bare $N$-point vertex functions with $L$ "insertions" of the squared field $\Phi_{0}^{2}$, the following definitions stand (for $d<4$ )

$$
\begin{aligned}
Z_{3}^{-1} & =\frac{\partial}{\partial p^{2}} \Gamma_{0}^{(0,2)}\left(p ; \bar{m}, g_{0}, d\right) \\
Z_{1}^{-1} & =\frac{\Gamma_{0}^{(0,4)}\left(\{0\} ; \bar{m}, g_{0}, d\right)}{g_{0}} \\
Z_{2}^{-1} & =\Gamma_{0}^{(1,2)}\left(\{0\} ; \bar{m}, g_{0}, d\right)
\end{aligned}
$$

in which $Z_{2}$ is a renormalization function which restores the linear measure of the distance to the critical temperature $T_{c}$, originally defined by the bare parameter $r_{0}$ and which has been lost when introducing the renormalized mass $\bar{m}$ [via Eq. (2) and a condition, not written here, which defines $\bar{m}$ as the inverse correlation length].

Since $\Lambda$ has been eliminated in the renormalization process, $\bar{m}$ plays the role of the effective wave-vector scale of reference and could be used as the unit to express the dimension of any dimensioned quantity. However, because $\bar{m}$ (the inverse of the correlation length) vanishes at the critical point it is preferable to use $g_{0}$ which, for $d \neq 4$, is a dimensioned constant at the critical temperature [see Eq. (10) below]. Hence, $\left(g_{0}\right)^{\frac{1}{4-d}}$ will be substituted to $\Lambda$ to play the role of the wave-vector unit (see below). Notice that in three dimensions $g_{0}$ has exactly the dimension of $\Lambda$ (i.e. of the inverse of a length) which is very convenient but not essential.

The dimensionless renormalization functions $Z_{i}$ have been calculated up to sixth order [24] and then partly up to seventh order [25] in powers of $g$. These series have been summed to estimate the critical exponents with great accuracy [26, 27] and also to determine nonasymptotic critical functions in the homogeneous phase [4, 5]. With the calculation of supplementary integrals [6], the calculations have been exended to the inhomogeneous phase up to fifth order allowing the determination of the nonasymptotic critical functions [6] in this phase and of the equation of state [27, 28]. The calculations of the 
nonasymptotic critical functions have been revisited 14] in order to account for the most recent estimates of the asymptotic universal critical quantities [27] and also to provide complete classical-to-critical crossover forms 14] which we are presently interested in.

\subsection{The crossover functions}

In the perturbative framework, the renormalized coupling $g$ may take on any value in the range $\left[0, g^{*}\right]$ where $g^{*}$ is its $\mathrm{W}-\mathrm{FP}$ value (a value which may be estimated in three dimensions by looking at the nontrivial zero of some series) and 0 is its G-FP value. The crossover functions have been obtained by resumming the series of a physical property $P$ of interest such as the correlation length $\ell(g)\left[=(\bar{m})^{-1}\right]$ or the susceptibily $\chi(g)$, for a discretized variation of $g$ in this range. An expression for the critical scaling field $t(g)$ (which "measures" the physical distance to the critical point) is obtained via an integration of the resummed series of $Z_{2}(g)$ (see ref. [5] for example). Hence the variations of $\ell$ or $\chi$, in terms of $t$, are primarily obtained implicitly via the dummy parameter $g$. Explicit functions of $t$ representing $\ell$ or $\chi$ are then obtained by fitting ad hoc forms to their discretized evolutions in the range $] 0, g^{*}$.

It is to be noted that, appart from $r_{0}$ which is used to determine $t$ (see below), the only remaining dimensioned parameter of the $\Phi_{d}^{4}$ model is the bare coupling $g_{0}$ with a dimension (in length unit) equal to $d-4$. Then, all the final functions are reduced by the appropriate powers of $g_{0}$ so as to be dimensionless.

In MR6, contrary to MR7, the entire crossover (corresponding to the complete range $] 0, g^{*}[$ ) had not been published. Also, in MR6, the error analysis was made independently from that associated with the estimates of the critical exponents done by Le Guillou and ZinnJustin [26]. In MR7 the convergence criteria for the Borel resummation of the different functions, like $\ell$ or $\chi$, was chosen such that the $\max$ and $\min$ bounds of the resulting critical exponents agreed as closely as possible to the (revised) values obtained by Guida and Zinn-Justin [27]. However, in doing so, it is more than likely that the error bars have been over estimated compared to what the resummation method used would have naturally indicated (following the rules applied in MR6). This is why the results of MR6 are also of interest to us especially in the case of the order parameter in the heterogeneous domain for which the large uncertainties of MR7 are not favourable for accurate fitting of the experimental data (see appendix B).

In MR7, two tables were presented to give an envelope for each function $F_{P}\left(t^{*}\right)$ representing a (dimensionless) model property $P$ versus a discretized dimensionless scaling field $t^{*}$ [defined in Eq. (11) below] over the entire range $] 0, g^{*}$ [ for the $\max$ and $\min$ bounds. The following expressions $F_{P}\left(t^{*}\right)$ were used to continuously fit the discrete data from each particular table

$$
F_{P}\left(t^{*}\right)=\mathbb{Z}_{P}^{ \pm}\left(t^{*}\right)^{-e_{P}} \prod_{i=1}^{K}\left(1+X_{P, i}^{ \pm}\left(t^{*}\right)^{D_{P}^{ \pm}\left(t^{*}\right)}\right)^{Y_{P, i}^{ \pm}}
$$

with

$$
D_{P}^{ \pm}\left(t^{*}\right)=\Delta-1+\frac{S_{P, 1}^{ \pm} \sqrt{t^{*}}+1}{S_{P, 2}^{ \pm} \sqrt{t^{*}}+1}
$$

In Eq. (5), $3 \leq K \leq 5$, depending on the required fit quality for each property $P=\left\{\left(\chi^{*}\right)^{-1} ;\left(\ell^{*}\right)^{-1} ; C^{*} ; m^{*}\right\}$ [the superscript $*$ indicates dimensionless quantities, $\chi^{*}$ is the susceptibility; $\ell^{*}$ is the correlation length; $C^{*}$ is the heat capacity; $m^{*}$ is the order parameter in the nonhomogeneous domain (note the distinction with the decorated $\bar{m}$ used for the renormalized mass)]. $e_{P}$ and $\Delta$ are the leading and first confluent universal exponents, respectively. The symbol \pm indicates the possible homogeneous $(+)$ and nonhomogeneous $(-)$ domains. All the constants $\mathbb{Z}_{P}^{ \pm}, X_{P, i}^{ \pm}, Y_{P, i}^{ \pm}$, and $S_{P, i}^{ \pm}$in Eqs. (5) and (6) are tabulated in [14]. They result from the fitting of eqs. (5) 6) to the theoretical calculations done point by point in the discretized complete $t^{*}=\{\infty, 0\}$ range corresponding to the complete range $] 0, g^{*}[$. The critical behavior of the specific heat is particular such that, compared to Eq. (5), it involves an additive critical constant that we note $X_{P, 6}^{ \pm}$.

\subsection{Physical validity of the functions}

\subsubsection{Analytical corrections discarded}

To compare the theoretical functions to measurements, we must relate the Hamiltonian parameters $\left(r_{0}, g_{0}, h\right)$ to their physical counterparts. This is done by the (usual) basic assumption that the bare quantities $\left(g_{0}, r_{0}, h\right)$ are analytical functions of the corresponding physical quantities $T$ and $H$ (in usual notations for magnetic systems) which control the approach to the actual critical point. Implicitly, we admit that the Hamiltonian energy is comparable to the physical free energy measured in unit of $k_{B} T \cong k_{B} T_{c}$ very close to the actual critical temperature $T_{c}$ of the system.

Assuming that analytical corrections to scaling are negligible, this introduces two arbitrary scale factors (noted $\vartheta$ and $\psi$ in the following) associated with the two bare fields $r_{0}$ and $h$, respectively, and one constant inverse length scale $\left(g_{0}\right)^{\frac{1}{4-d}}$ which fixes the dimensionality of the physical variables. This can be accomplished as follows:

1. At $h=0$, the bare field $r_{0}=r_{0}(T)$ must be related to the actual temperature $T$ of the system, leading to define its critical value $r_{0 c}=r_{0}\left(T_{c}\right)$ from the actual critical temperature $T_{c}$ of the system. For $T$ 
close to $T_{c}$, it becomes possible to relate the bare field difference $r_{0}(T)-r_{0 c}\left(T_{c}\right)$ to the temperature distance $T-T_{c}$ by the following analytical (linear) approximation

$$
r_{0}-r_{0 c}=\mathrm{const} \times\left(T-T_{c}\right)+\mathcal{O}\left[\left(T-T_{c}\right)^{2}\right]
$$

In an equivalent manner, at $r_{0}=r_{0 c}$, the bare field $h$ must be related to the actual (magnetic field) variable $H$ (in notations for magnetic system) of the system, leading also to the possible analytical (linear) approximation

$$
h=\text { const } \times H+\mathcal{O}\left[H^{2}\right]
$$

for $h$ close to zero. Correlatively, the linearization between the bare order parameter $m=\left\langle\Phi_{0}\right\rangle$ to the actual (magnetization) density variable $M$ (in notations for magnetic system) reads

$$
\begin{aligned}
m= & \text { const } \times M\left[\left(T-T_{c}\right), H\right]+ \\
& \mathcal{O}\left[\left(T-T_{c}\right)^{2}, H^{2}, H \times\left(T-T_{c}\right)\right]
\end{aligned}
$$

where the two constant prefactors of Eqs. (8) and (9) are interrelated by thermodynamic considerations attached to the conjugated variables $M$ and $H$ (or $m$ and $h$ equivalently).

Finally, at the above linearized order of the relations between the bare fields and the physical fields, close to the critical point defined by $T-T_{c}=0$ (or $r_{0}-r_{0 c}=0$ ) and $H=0$ (or $h=0$ ), we can introduce one finite value of the coupling constant such as,

$$
g_{0}=\text { const }+\mathcal{O}\left(T-T_{c}\right)+\mathcal{O}[H]
$$

which appears then as a system-dependent critical quantity which must take the $d-4$ dimension (in length unit). In the Eqs. (7) to (9), one generally keeps only the leading terms but one must keep in mind that far away from $T_{c}$, the neglected second order analytical terms $\mathcal{O}\left[\left(T-T_{c}\right)^{2}\right], \mathcal{O}\left[H^{2}\right]$, and $\mathcal{O}\left[H \times\left(T-T_{c}\right)\right]$ (within $r_{0}, h$ and $m$ ) could have some importance especially in approaching the GFP.

2. With $g_{0}$ defined by Eq. (10), we can now appropriately make dimensionless the bare quantity $r_{0}$ by introducing the dimensionless scaling field $t^{*}$ used in Eqs. (5) and (6):

$$
t^{*}=\frac{r_{0}-r_{0 c}}{\left(g_{0}\right)^{\frac{2}{4-d}}}
$$

For the actual system, it is convenient to define the reduced temperature distance in units of the critical temperature (already chosen to express the energy unit of the hamiltonian), leading to the usual notation of the thermal like field $\Delta \tau^{*}=\frac{T-T_{c}}{T_{c}}$. Assuming a "sufficiently" small $\Delta \tau^{*}, t^{*}$ and $\Delta \tau^{*}$ are related by the first dimensionless arbitrary scale factor $\vartheta$ :

$$
t^{*}=\vartheta \times \Delta \tau^{*}
$$

Similar assumptions stand for the second scaling field $h$ which must be related to $H$ by Eq. (8).

A second arbitrary scale factor appears, noted $\psi$, between the dimensionless field $h^{*}=\frac{h}{\left(g_{0}\right)^{\frac{d+2}{2(4-d)}}}$ and its corresponding dimensionless physical quantity $H^{*}$ (here in notations for magnetic systems):

$$
h^{*}=\psi \times H^{*}
$$

Correspondingly, the dimensionless order parameter field $m^{*}=\left\langle\Phi_{0}^{*}\right\rangle=\frac{m}{\left(g_{0}\right)^{\frac{d-2}{2(4-d)}}}$ is then related to the dimensionless physical quantity $M^{*}$ (here in notation for magnetic systems) by

$$
m^{*}=(\psi)^{-1} \times M^{*}
$$

Finally the "length" $\left(g_{0}\right)^{\frac{1}{d-4}}$ must be related to a microscopic length scale $a$ characteristic of the actual system as:

$$
\left(g_{0}\right)^{\frac{1}{d-4}}=u_{0}^{*} \times a
$$

where $u_{0}^{*}$ is a dimensionless number similar to $\vartheta$ and $\psi$. Notice that $u_{0}^{*}$ has the characteristic of taking a value which depends on the (a priori) choice of a system dependent length scale $a$, not attached to the critical behavior. That characteristic will be used to determine a subclass of "comparable" systems [i.e. systems having a comparable characteristic length scale $a$ (see below)].

As will be shown in Appendix A, it is worthwhile already indicating here that the consideration of $a$ via Eq. 15 is not required when the correlation length is considered alone. This is because the dimensionless correlation length $\ell^{*}$ of the theoretical model is naturally compared with the experimental measurement $\xi$ via the following relation:

$$
\ell^{*}=\left(g_{0}\right)^{\frac{1}{4-d}} \times \xi
$$

In Appendix A, limiting our calculations to the $d=3$ case, we illustrate the way the adjustable parameters are determined where the emphasis is also on the choice of the microscopic length scale $a$ appearing in Eq. (15). When the critical temperature of the system is known, fitting the asymptotic two-term expansion of our theoretical crossover functions to the singular behavior of the experimental quantities, permits unambiguous determinations of the three system-dependent parameters $\vartheta, \psi$, and $g_{0}$. We then indicate how the dimensionless coupling 
constant $u_{0}^{*}$ may be used to characterize "comparable" systems which belong to a same subclass of universality when an explicit length scale unit $(a)$ is known for each system. We show that the theoretical crossover functions then provide an explicit analytical form of the "master" (i.e. unique) singular behavior associated to this subclass and discriminate the role of the energy and length scale factors for the subclass.

\subsubsection{Non-analytical corrections discarded - Some ideas on renormalization}

The renormalized $\Phi_{d}^{4}(n)$ model of the perturbative FT is an efficient reduction of a complicated mathematical problem which originally involves an infinite number of parameters to one parameter $g$ (the renormalized $\Phi^{4}$ coupling $g$ of section 2.11). In order to better understand the impact of this reduction, it is necessary to consider the complete and non-perturbative renormalization group theory developed by Wilson [3].

Explaining the use of the renormalization theory in the study of critical phenomena is out of the scope of the present paper. However, it is worthwhile to indicate briefly some general ideas which may help someone to understand the use of our crossover functions in comparison with experimental data.

The RG theory is a general theory to treat situations where infinitely many degrees of freedom are correlated, such as near a critical point where the correlation length $\xi$ diverges. Since in such cases, we are essentially interested in describing the large distance behavior, and because of the correlations, one may represent the state of a system near criticality by means of a dimensionless hamiltonian $\mathcal{H}$ which depends only on a local field $\phi(x)$ which summarizes, over a volume of linear size $\Lambda^{-1}$ centered on $x$, only general properties of the genuine local variable which critically fluctuates (e.g. the spin variables $S_{i}$ of the Ising system). Beyond $\phi(x), \mathcal{H}$ depends also on the relevant physical parameters like the temperature $T$ and the magnetic field $H$ (for the sake of simplicity we shall not consider $H$ ). The form of $\mathcal{H}$ is quite general provided it satisfies some required properties of symmetry. For systems which are $O(1)$-symmetric [more precisely $Z_{2}$-symmetric, i.e. invariant under the change $\phi \rightarrow-\phi$ such as Ising-like systems], then $\mathcal{H}$ must be an even function of $\phi$ (when $H=0$ ).

In order to concretize a bit the form of $\mathcal{H}$, one may look at its expansion for small values of $\phi(x)$, in the case of the $Z_{2}$-symmetry, it becomes ( $a_{0}$ being a term which is usually discarded in FT) :

$$
\begin{aligned}
\mathcal{H}= & a_{0}+\int d^{d} x\left\{a_{1}(\nabla \phi)^{2}+a_{2} \phi^{2}+a_{3} \phi^{4}\right. \\
& \left.+a_{4} \phi^{6}+a_{5} \phi^{2}(\nabla \phi)^{2}+\cdots\right\}
\end{aligned}
$$

in which the expansion in powers of the derivatives is a consequence of the short range interactions between the original spins and the coefficients $a_{i}$ depend on $T$. Actually one is free to redefine the global normalization of the field so as to set $a_{1}=$ constant (in general one chooses $a_{1}=\frac{1}{2}$ ) but this is not mandatory. Furthermore all the dimensions are measured in terms of $\Lambda$, so that it is convenient to deal with dimensionless quantities in $\mathcal{H}$. The set $\left\{a_{i}\left(\frac{T}{T_{c}}\right)\right\}$ characterizes a given physical system near a critical temperature $T_{c}$. It may be seen as the coordinate of a point in a space $\mathcal{S}$ of infinite dimension.

The problem is that calculation of any critical quantity with $\mathcal{H}$ is very complicated. The reason is that the parameters at hand $\left\{a_{i}\left(\frac{T}{T_{c}}\right)\right\}$ are attached to the length scale $\Lambda^{-1}$, while the physics under consideration refers to phenomena that occur at all the length scales smaller than $\xi$ and greater than $\Lambda^{-1}$. Now because the ratio $\frac{\xi}{\Lambda^{-1}}$ tends to infinity, there are an infinity of scales to be accounted for to solve the problem.

Introducing a parameter $s$ such as $s \in[0, \infty[$, the renormalization group transformations precisely construct successive hamiltonians $\mathcal{H}(s)$, obtained by integrating out the degrees of freedom over the scales ranging in $\left[\left(e^{-s} \Lambda\right)^{-1}, \Lambda^{-1}\right]$, and then rescaling back the wavevector scale (hence $\Lambda^{\prime}=e^{-s} \Lambda \rightarrow \Lambda$ ). By construction, the renormalized hamiltonian $\mathcal{H}(s)$ presents a correlation length $\xi_{s}$ which is reduced, compared to the $\xi$ of the initial hamiltonian $\mathcal{H}(s=0)=\mathcal{H}$, with $\xi_{s}=e^{-s} \xi$, and the effective hamiltonian $\mathcal{H}(s)$ is no longer critical when $\frac{\xi}{\Lambda^{-1}} \simeq 1$. If the initial hamiltonian is critical (i.e. if $\xi=\infty)$, then $\mathcal{H}(s)$ reaches a fixed point when $s \rightarrow \infty$.

The evolution of $\mathcal{H}(s)$ under an infinitesimal change of $s$ is governed by an equation, called the exact RG equation. It is a complicated integro-differential equation which may be expanded in powers of the derivative of the field without losing the non-perturbative character of the complete theory (for a review see [29]).

At leading order of the derivative expansion, called the local potential approximation (LPA), the exact RG equation reduces to an ordinary differential equation for a function $V$ of one variable $\phi$. Its relation to the $\Phi_{d}^{4}$ model is better seen if one expands $V$ in powers of $\phi$. Imposing the property of parity in $\phi$, it becomes (for $\phi$ close to 0 ):

$$
V(\phi, s)=V_{0}(s)+V_{2}(s) \phi^{2}+V_{4}(s) \phi^{4}+V_{6}(s) \phi^{6}+\cdots
$$

The form of Eq. (18) shows the relation with Eq. (11) when the derivatives of the field are neglected (again, for the sake of simplicity, the account of $h$, which would require the presence of odd powers of $\phi$, is not considered here). In particular, the initial form chosen for $V$ may be directly related to the bare parameters of the $\Phi_{d}^{4}$ model with, e.g. $\Lambda^{2} V_{2}(0)=\frac{r_{0}}{2}$, and $\Lambda^{4-d} V_{4}(0)=\frac{g_{0}}{4 !}$ (and $\left.V_{0}(0)=V_{6}(0)=0\right)$, they are parameters attached to the microscopic length scale $\Lambda^{-1}$ of the initial hamiltonian $(s=0)$. As for the renormalized coupling $g$ of the perturbative framework, it is not a constant but a running parameter like $V_{4}(s)$. To be more precise, one must look at the flow of $V(\phi, s)$ solution of the RG equation. 
In LPA, the derivatives of the field are neglected but one may account for all the effects of all the Hamiltonian terms via the evolution of the complete function $V(\phi)$ under the action of the RG transformation.

In three dimensions, a non-trivial fixed point $\stackrel{\circ}{V}^{\prime}(\phi)$ exists and may be (numerically) determined. One may illustrate this by writing (as a formal polynomial of $\phi$ ):

$$
\stackrel{\circ}{V}(\phi)=\stackrel{\circ}{V}_{0}+\stackrel{\circ}{V}_{2} \phi^{2}+\stackrel{\circ}{V}_{4} \phi^{4}+\stackrel{\circ}{V}_{6} \phi^{6}+\cdots
$$

and then calculating the values of $\stackrel{\circ}{V}_{0}, \stackrel{\circ}{V}_{2}, \stackrel{\circ}{V}_{4}, \stackrel{\circ}{V}_{6}$, etc. As a consequence, one may situate the fixed point in the formal space $\mathcal{S}$ truncated to the set of couplings $\left\{V_{0}, V_{2}, V_{4}, V_{6}, \cdots\right\}$ of infinite dimension. To visualize the evolution of a RG trajectory of $V(\phi, s)$ (the evolution of $V(\phi)$ under a continuous RG transformation controled by the parameter $s \in[0, \infty[$ in $\mathcal{S})$, one may project it in the plane $\left\{V_{4}, V_{6}\right\}$, for example.

We may choose to integrate the differential equation with an initial simple form for $V(\phi, s=0)$ like, for example:

$$
V(\phi, 0)=V_{2}(0) \phi^{2}+V_{4}(0) \phi^{4}+V_{6}(0) \phi^{6}
$$

in which $V_{2}(0), V_{4}(0)$ and $V_{6}(0)$ are numbers representing the initial values of $V(s)$. As soon as $s \neq 0$, then $V(\phi, s)$ contains all powers of $\phi$.

To approach the fixed point $\stackrel{\circ}{V}(\phi)$ starting with (20), one must adjust one of the three initial parameters, e.g. $V_{2}(0)$, to a particular value $V_{2 c}$, which depends on the value chosen for the other parameters $V_{4}(0)$ and $V_{6}(0)$. The requirement is similar to the adjustment of the critical temperature with a view to reach the critical point in actual systems. The resulting value $V_{c}(\phi)$ is said to belong to the critical subspace $\mathcal{S}_{c}$ of $\mathcal{S}$, which forms the domain of attraction to the W-FP. $\mathcal{S}_{c}$ has the same dimension as $\mathcal{S}$ minus one (the codimension of $\mathcal{S}_{c}$ is equal to one) which corresponds to the fact that the W-FP has only one direction of unstability, corresponding to a direction locally orthogonal to $\mathcal{S}_{c}$ (we suppose $h=0$ ).

It is thus possible to illustrate the RG trajectories in $\mathcal{S}_{c}$ which, starting from some arbitrary points of $\mathcal{S}$ reach the W-FP when $s \rightarrow \infty$ (one initial parameter must be finely adjusted). This is demonstrated in Figure 1. On this figure one sees that, whatever their starting points, the critical trajectories reach the W-FP asymptotically along a unique ideal trajectory which links the G-FP to the W-FP. The closer the starting points are chosen to the G-FP, the longer is the way along this ideal trajectory. This ideal RG trajectory is called the "renormalized trajectory" (RT) because the RG flow running on it corresponds, in the vicinity of the G-FP, to the RG flow discovered in the perturbative theory of renormalization. Since the RT is a manifold of dimension one, a single parameter (the renormalized coupling "constant" $g$ ) is sufficient to characterize the RG flow running on it.

Hence, in some sense (there is an arbitrariness in the definition of the renormalized coupling) the renormalized (or running) coupling $g$ of the perturbative framework

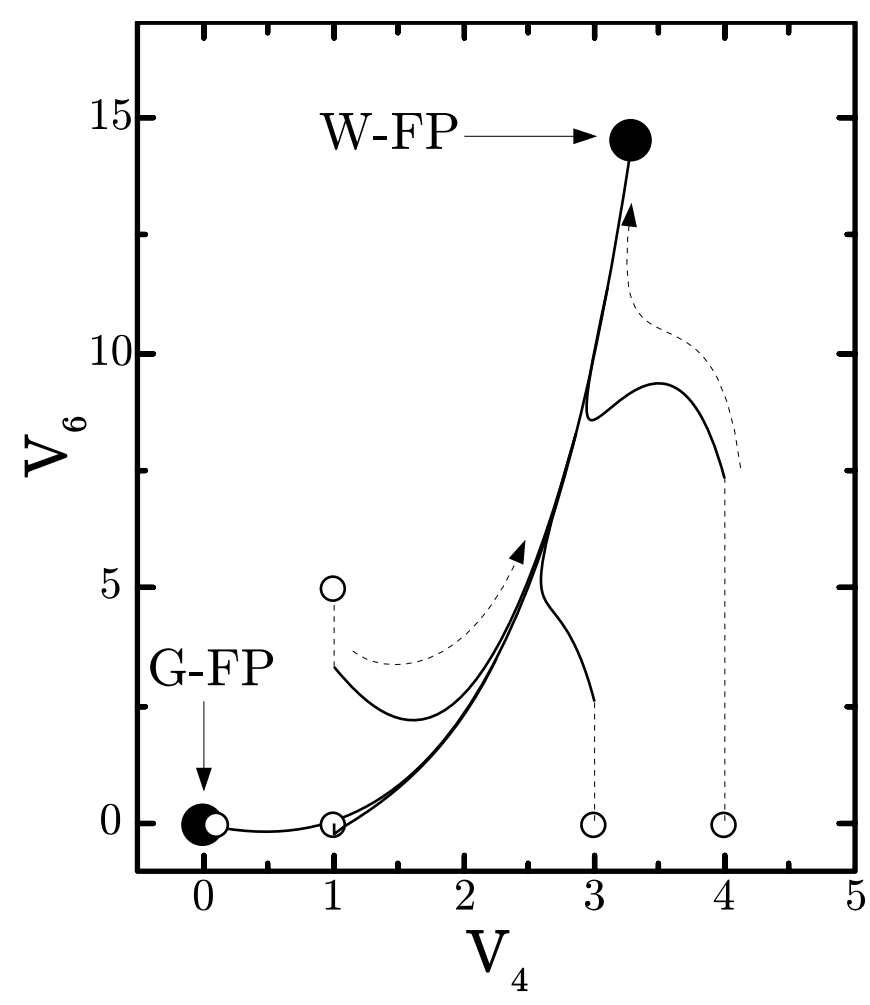

Figure 1: Ideal Renormalization Group trajectory in the binary diagram of coupling parameters $V_{4}$ and $V_{6}$ [see text and Eqs. (19) and (20)].

implicitly "flows" along the RT. Actually, $g$ is a function of $s$, more precisely in the circumstances of the massive field theory, it is a function of $\frac{\bar{m}}{\Lambda}$. The difference with the complete Wilson's RG theory is that, in the perturbative framework, one has implicitly assumed to be "exactly on" the RT, so that the distance to the W-FP is measured by the values taken by a unique parameter $g\left(\frac{\bar{m}}{\Lambda}\right)-g^{*}\left(g^{*}\right.$ being the value of $g$ at the $\mathrm{W}-\mathrm{FP})$. The consequence is the presence of only one family of correction-to-scaling terms, such as in the following expansion of the (dimensioless) correlation length for example:

$$
\ell^{*}\left(t^{*}\right)=\ell_{0}^{* \pm}\left|t^{*}\right|^{-\nu}\left[1+\sum_{k=1}^{\infty} b_{k}^{ \pm}\left|t^{*}\right|^{k \Delta}\right]
$$

with $\Delta$ a specific exponent the value of which (close to $0.5)$ may be estimated by resumming series of the renormalized perturbation theory of the $\Phi_{d}^{4}$ model. It is precisely expansions like in Eq. (21) that have been summed into the crossover functions considered above.

The other transients not accounted for by the $\Phi_{d}^{4}$ model (by implicitly assuming that the only possible RG trajectory is the RT) are responsible for other kinds of correction-to-scaling terms characterized by a hierachical set of exponents $\Delta_{j}(j=2, \cdots, \infty)$ such that:

$$
\Delta<\Delta_{2}<\Delta_{3}<\cdots<\Delta_{\infty}
$$


The fact that $\Delta$ (the exponent which controls the asymptotic approach, along the RT, to the W-FP) is the smallest exponent is well illustrated on Figure 1 by the coincidence of all trajectories with the RT before reaching the W-FP.

The few estimates of $\Delta_{2}$ indicate that its value in three dimensions and for $n=1$ is of order $2 \Delta$ so that, on a general ground, the expansion (21) is a priori not valid beyond the first correction term. This is why one often uses the following two-term Wegner expansion to analyse the experimental data:

$$
\xi\left(\Delta \tau^{*}\right)=\xi_{0}^{ \pm}\left|\Delta \tau^{*}\right|^{-\nu}\left[1+a_{\xi}^{ \pm}\left|\Delta \tau^{*}\right|^{\Delta}\right]
$$

with the exponents $\nu$ and $\Delta$ fixed to their FT values. The domain of validity of such a two-term expansion is called the preasymptotic domain (PAD) and noted $\left|t^{*}\right| \lesssim$ $\mathcal{L}_{P A D}^{I s i n g}$

For the same reason, the crossover functions calculated in FT were not expected to generally reproduce the experimental data beyond the Ising like PAD. However this objection does not account for the amplitudes of the correction terms. It may well occur that the coefficients of the corrections associated to the exponents $\Delta_{j}(j=2, \cdots, \infty)$ are very small so that in effect only the $\Phi_{d}^{4}$-like corrections have to be considered even on a range of $t^{*}$ for which several (more than two) terms of the expansion of Eq. (21) are not negligible. It is the case of initial Hamiltonians the coordinates of the critical point of which correspond to a point lying very close to the RT. This particularity may even validate the entire crossover functions of FT if the initial point lies close to the G-FP.

Of course, the RT does not represent the unique possibility of approaching to the W-FP and the infinite dimension of $\mathcal{S}_{c}$ leaves room for an infinity of possibilities. For example, there exists another attractive trajectory which is characterized by an asymptotic approach to the W-FP controlled by $\Delta_{2}$, and supplementary adjustment of the initial Hamiltonian is needed to obtain this kind of approach. For systems represented by such Hamiltonians, the crossover functions of FT have no utility at all since none of the correction-to-scaling terms is correct. Similar and more common are those systems which correspond to an approach to the W-FP from the "opposite side" compared to the RT. Their critical behavior are characterized by amplitudes of the first correctionto-scaling (controlled by $\Delta$ ) with a sign opposite to that generated by the approach along the RT [30].

Up to now, it is not possible to say a priori which kind of approach to the W-FP may correspond to an actual system which belongs to a given universality class. A way to find out is to try a fit of test functions to experimental data. From such tests, it seems that the subclass of onecomponent fluids corresponds to Hamiltonian lying very close to the RT [31]. This is why the crossover functions of FT are good candidates to fit the scaling curves of that subclass.

\section{THE THEORETICAL MEAN CROSSOVER FUNCTIONS}

In this section, we determine mean values of the crossover functions. We also take the opportunity of simplifying the ad hoc functions by limiting the products in Eq. (5) to only three terms. Hence we have to determine the value of parameters like those entering Eqs. (5) and (6), but with $K=3$ for all quantities and so that the corresponding ad hoc functions lie just in-between the max and min bounded functions published in Ref. [14]. To accomplish this, the following is taken into consideration.

1. The required precision in fitting each property requires the recourse to phenomenological confluent functions $D_{p}^{ \pm}\left(t^{*}\right)$ [Eq. [6]] which essentially account for the crossover between the values $\Delta \sim \frac{1}{2}$ of the Ising-like confluent exponent as $t^{*} \rightarrow 0$, and the exact value $\Delta_{m f}=\frac{1}{2}$ of the mean-field-like "confluent" exponent as $t^{*} \rightarrow \infty$. Such a crossover introduces the following condition

$$
S_{P, 1}^{ \pm}=S_{P, 2}^{ \pm}\left(1-\Delta+\Delta_{m f}\right)
$$

which confers to one parameter among $S_{P, 1}^{ \pm}$and $S_{P, 2}^{ \pm}$, a noticeable difference from the other constants appearing in Eqs. (5) and (6).

2. In [14], the ad hoc functions for the susceptibility in the homogeneous phase are already defined with a three-term product. In that case, the behaviors of $D_{\chi}^{+}\left(t^{*}\right)$ as a function of $t^{*}$ as illustrated in Figure 2] clearly show that the condition

$$
S_{\chi, 2}^{ \pm} \sqrt{t_{0}^{*}}=1
$$

can be used as an indicative sensor of the classicalto-critical crossover (C3) domain ( $t_{0}^{*}$ stands for the values of $t^{*}$ where the ad hoc confluent functions take on precisely the mean value $\Delta_{\frac{1}{2}}=\frac{\Delta+\Delta_{m f}}{2}$ ). The extension $\delta \mathcal{L}_{C 3}$ of the associated "intermediate" $t^{*}$-range can be measured by a characteristic amplitude $\varpi<1$ [which remains to be defined, see below Eq. [39]], such that to $\varpi\left(S_{\chi, 2}^{ \pm}\right)^{-2} \lesssim$ $t^{*} \in\left[\delta \mathcal{L}_{C 3}\right] \lesssim \frac{1}{\varpi}\left(S_{\chi, 2}^{ \pm}\right)^{-2}$, separating thus unambiguously an Ising-like asymptotic domain of extension $t^{*}<\mathcal{L}^{I \operatorname{sing}}=\varpi\left(S_{\chi, 2}^{ \pm}\right)^{-2}$ and a mean-field like asymptotic domain of extension $t^{*}>\mathcal{L}^{m f}=$ $\frac{1}{\varpi}\left(S_{\chi, 2}^{ \pm}\right)^{-2}$ (see Figure 2).

3. The leading amplitudes $\mathbb{Z}_{P}^{ \pm}$, associated with their respective universal exponents $e_{P}$ satisfying scaling laws, are unambiguously related by universal amplitude combinations between them.

4. A part of $\left\{X_{i}, Y_{i}\right\}_{P}^{ \pm}$'s accounts for the universal features associated to the critical confluent corrections 


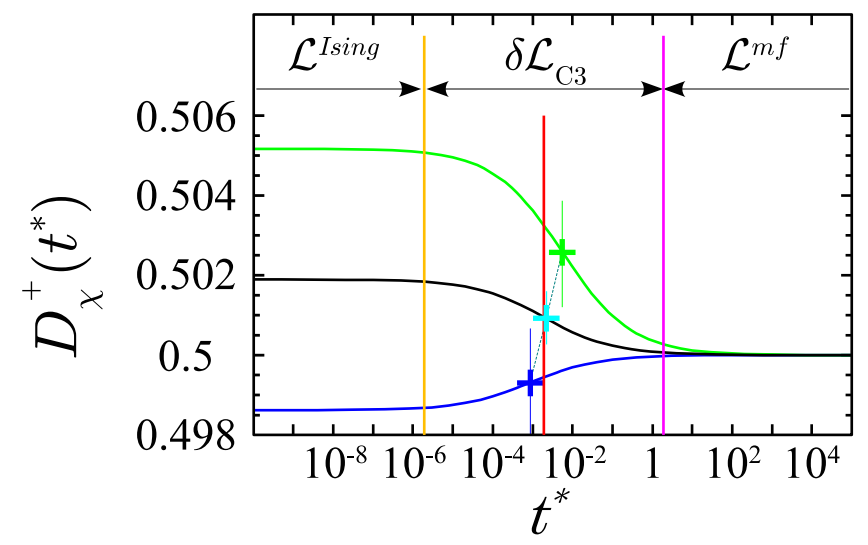

Figure 2: Typical crossover behaviors of the confluent crossover function $D_{\chi}^{+}\left(t^{*}\right)$ [Eq. [6)], for the bounded (max and $\mathrm{min}$ ) critical confluent exponents. as a function of the thermal field (at zero ordering field) for the susceptibility in the homogenous phase $\left(T>T_{c}\right)$. The vertical lines are indicative of typical discrete variations of the confluent function, showing the practical interest of the condition $S_{\chi, 2}^{+}\left(\Delta, \Delta_{m f}\right) \sqrt{t_{0}^{*}}=1$ (crosses), which corresponds to $D_{\chi}^{+}\left(t_{0}^{*}\right)=\Delta_{\frac{1}{2}}=\frac{\Delta+\Delta_{m f}}{2}$. Using a single perfactor $\varpi<1$ which remains to be estimated [see below Eq. [40]], it is possible to define the extensions $t^{*}<\mathcal{L}^{I \text { sing }}=\varpi\left(S_{\chi, 2}^{+}\right)^{-2}$, $t^{*}>\mathcal{L}^{m f}=\frac{1}{\varpi}\left(S_{\chi, 2}^{+}\right)^{-2}$, and $\mathcal{L}^{I s i n g} \lesssim t^{*} \in\left[\delta \mathcal{L}_{C 3}\right] \lesssim \mathcal{L}^{m f}$, of either, the Ising-like (Ising), or the mean-field-like $(m f)$, or the classical-to-critical crossover $(C 3)$ domains, respectively (see text for details).

to scaling, explicitly characterized by the single universal exponent $\Delta$ and one first confluent amplitude (see below) for $t^{*} \rightarrow 0$.

5. Another part accounts for the asymptotic meanfield behavior for $t^{*} \rightarrow \infty$ [implicitly characterized by the two (leading and confluent) exponents $e_{P, m f}$ and $\Delta_{m f}$ and their associated amplitudes (see below)].

6. The remaining part accounts for the expected classical-to-critical crossover (C3) in the intermediate $t^{*}$-range (which also remains to be defined, see point 2 just above).

These remarks provide constraints on the parameter entering each specific ad hoc functions. Let us consider those constraints explicitly.

\subsection{D Ising-like PAD description}

Since the error estimates are the largest in the vicinity of the W-FP, it is in the Ising-like PAD limit that the determination of the mean value has important consequences.

Let us define a set of four constraints to control the mean Ising-like PAD description within the two limiting
Ising-like PAD descriptions given by the bounded functions $F_{P, \max }$ and $F_{P, \min }$, respectively.

Starting with Eq. (5), we first consider the two-term (Wegner) expansion of $F_{P}\left(t^{*}\right)$ :

$$
F_{P, I s i n g}^{P A D}\left(t^{*}\right)=\mathbb{Z}_{P}^{ \pm}\left(t^{*}\right)^{-e_{P}}\left[1+\mathbb{Z}_{P}^{1, \pm}\left(t^{*}\right)^{\Delta}\right]
$$

where the function $F_{P, I s i n g}^{P A D}\left(t^{*}\right)$ is valid within some Ising-like PAD extension $t^{*} \leq \mathcal{L}_{P A D}^{\text {Ising }}$. To estimate mean exponents and mean amplitudes of Eq. (24) from max and min ones, we impose the following four obvious conditions

$$
\begin{array}{r}
e_{P}=\frac{e_{P, \max }+e_{P, \text { min }}}{2} \\
\mathbb{Z}_{P}^{ \pm}=\sqrt{\mathbb{Z}_{P, \max }^{ \pm} \mathbb{Z}_{P, \text { min }}^{ \pm}} \\
\Delta=\frac{\Delta_{\max }+\Delta_{\text {min }}}{2} \\
\mathbb{Z}_{P}^{1, \pm}=\sum_{i=1}^{3} X_{P, i}^{ \pm} Y_{P, i}^{ \pm} \\
=\sum_{i=1}^{K} \frac{X_{P, \text { max }, i}^{ \pm} Y_{P, \text { max }, i}^{ \pm}+X_{P, \text { min }, i}^{ \pm} Y_{P, \min , i}^{ \pm}}{2}
\end{array}
$$

The leading term of our mean function is then identical to the leading term of the mixing function

$$
\begin{aligned}
F_{P, \text { mix }}^{E}\left[t^{*}, D_{P, \text { mix }}^{ \pm}\left(t^{*}\right)\right] & =\left\{F_{P, \text { max }}\left[t^{*}, D_{P, \text { max }}^{ \pm}\left(t^{*}\right)\right]\right\}^{E} \\
& \times\left\{F_{P, \text { min }}\left[t^{*}, D_{P, \text { max }}^{ \pm}\left(t^{*}\right)\right]\right\}^{1-E}
\end{aligned}
$$

proposed in [14], with $E=\frac{1}{2}$. The three first Eqs. (25), (26), and (27), provide unequivocal determination of the mean values of the three parameters $e_{P}, \mathbb{Z}_{P}^{ \pm}$, and $\Delta$, respectively. The Eq. (28) involves exclusively the $\left\{X_{i}, Y_{i}\right\}_{P}^{ \pm}$s.

\subsection{Mean-field-like PAD description}

To recover the (asymptotic) mean-field-like behavior of $F_{P}\left[t^{*}, D_{P}^{ \pm}\left(t^{*}\right)\right]$ in the limit $t^{*} \rightarrow \infty$, the following relations are required

$$
e_{P, m f}=e_{P}-\frac{1}{2} \sum_{i=1}^{3} Y_{P, i}^{ \pm}
$$

$$
\mathbb{Z}_{P, m f}^{ \pm}=\mathbb{Z}_{P}^{ \pm} \prod_{i=1}^{3}\left(X_{P, i}^{ \pm}\right)^{Y_{P, i}^{ \pm}}
$$

where $e_{P, m f}$ and $\mathbb{Z}_{P, m f}^{ \pm}$are the mean-field (classical) values of the asymptotic exponent and amplitude, respectively. Such a mean-field situation as $t^{*} \rightarrow \infty$, can be 
easily characterized in an equivalent manner to the above Ising situation as $t^{*} \rightarrow 0$.

Therefore, the following restricted two-term expansions

$$
F_{P, m f}^{P A D}\left(t^{*}\right)=\mathbb{Z}_{P, m f}^{ \pm}\left(t^{*}\right)^{-e_{m f}}\left[1+Z_{P, m f}^{1, \pm}\left(t^{*}\right)^{-\Delta_{m f}}\right]
$$

can be easily determined for $t^{*} \rightarrow \infty$, which are valid close to the G-FP, within the mean-field-like PAD extension $t^{*} \geq \mathcal{L}_{P A D}^{m f}$. In Eq. (32), $\Delta_{m f}$ is the mean field exponent of the first order term of "classical" corrections, and $\mathbb{Z}_{P, m f}^{1, \pm}$ is the associated amplitude defined by the following equation

$$
\mathbb{Z}_{P, m f}^{1, \pm}=\sum_{i=1}^{3} \frac{Y_{P, i}^{ \pm}}{X_{P, i}^{ \pm}}
$$

As in the case of the Ising-like PAD description, we impose four conditions on the ad hoc mean function to describe the mean-field PAD using the original pairs $\left\{X_{i}, Y_{i}\right\}_{P}^{ \pm}$'s of the bounded functions. These four conditions read as follows

$$
\begin{aligned}
& e_{P, m f}=e_{P}-\frac{1}{2} \sum_{i=1}^{3} Y_{P, i}^{ \pm} \\
&=\frac{1}{2}\left[e_{P, \max }+e_{P, \min }\right. \\
&\left.-\frac{1}{2} \sum_{i=1}^{K}\left(Y_{P, \max , i}^{ \pm}+Y_{P, \min , i}^{ \pm}\right]\right] \\
& \mathbb{Z}_{P, m f}^{ \pm}= \mathbb{Z}_{P}^{ \pm} \prod_{i=1}^{3}\left(X_{P, i}^{ \pm}\right)^{Y_{P, i}^{ \pm}} \\
&= \sqrt{\mathbb{Z}_{P, \max }^{ \pm} \mathbb{Z}_{P, \min }^{ \pm}} \\
& \times \prod_{i=1}^{K} \sqrt{\left(X_{P, \max , i}^{ \pm}\right)^{Y_{P, \max , i}^{ \pm}}\left(X_{P, \min , i}^{ \pm}\right)^{Y_{P, m i n}, i}} \\
& \Delta_{P, m f}^{ \pm}=\frac{1}{2} \\
& \mathbb{Z}_{P, m f}^{1, \pm}=\sum_{i=1}^{3} \frac{Y_{P, i}^{ \pm}}{X_{P, i}^{ \pm}} \\
&=\frac{\lambda_{P}}{2} \sum_{i=1}^{K}\left(\frac{Y_{P, \max , i}^{ \pm}}{X_{P, \max , i}^{ \pm}}+\frac{Y_{P, \min , i}^{ \pm}}{X_{P, \min , i}^{ \pm}}\right)
\end{aligned}
$$

Eq. (36) fixes the mean value of the mean-field-like "confluent" exponent, unequivocally. The three Eqs. (34), (35), and (37), added to Eq. (28), impose the mean values of two pairs among the 3 pairs $\left\{X_{i}, Y_{i}\right\}_{P}^{ \pm}$'s.

\subsection{Number and nature of the parameters}

The above analysis demonstrates that the theoretical mean function $F_{P}\left[t^{*}, D_{P}^{ \pm}\left(t^{*}\right)\right]$ must satisfy 9 constraints [Eqs. (23), (25) to (28), and (34) to (37)] to reproduce the two asymptotic and pre-asymptotic branches of a complete crossover effect. Therefore, any $F_{P}\left[t^{*}, D_{P}^{ \pm}\left(t^{*}\right)\right]$ must contain at least 9 parameters.

Eight of them can be readily unequivocally determined: $e_{P}$ from Eq. (25), $\mathbb{Z}_{P}^{ \pm}$from Eq. (26), $\Delta$ from Eq. (27), $\Delta_{m f}$ from Eq. (36) and two $\left\{X_{i}, Y_{i}\right\}_{P}^{ \pm}$pairs from the four Eqs. (28), (34), (35), and (37). Using $S_{P, 2}^{ \pm}\left(\Delta, \Delta_{m f}\right)$ as an entry data in Eq. (6), leads to the supplementary determination of $S_{P, 1}^{ \pm}\left(\Delta, \Delta_{m f}\right)$ from Eq. (23). Subsequently, the parameters attached to the intermediate part of the crossover need to be determined.

The phenomenological forms of Eqs. (5), (6) and (23), with $K=3$, introduce 12 parameters [the case with $K=$ 2 (10 parameters) is of limited interest due to the errorbar propagation which cannot be accounted for via only two $\left\{X_{i}, Y_{i}\right\}_{P}^{ \pm}$pairs]. Therefore, for any fitting procedure which uses $S_{P, 2}^{ \pm}\left(\Delta, \Delta_{m f}\right)$ as an entry (free) parameter, only one $\left\{X_{i}, Y_{i}\right\}_{P}^{ \pm}$pair, remains truly free in the $(K=3)$ product terms of the MR crossover function.

The triad $\left\{S_{2}, X_{i}, Y_{i}\right\}_{P}^{ \pm}$of the calculated parameters is composed of one "crossover sensor" $S_{P, 2}^{ \pm}$, characteristic of the $t^{*}$-location of the classical-to-critical confluent crossover (see Figure 2), and one "amplitudeexponent pair" $\left\{X_{i}, Y_{i}\right\}_{P}^{ \pm}$, proper to the shape of the strict crossover part between the two asymptotic PAD behaviors (we will illustrate this latter point below using Figure 4 in $\S 3.4$ ).

Let us look for the possible existence of a unique value $S_{2}=$ cte [or $S_{1}=$ cte by virtue of Eq. (23)], whatever the selected property and the considered (homogeneous or non-homogeneous) phase of the system.

The $S_{P, i}^{ \pm}\left(\Delta, \Delta_{m f}\right)$ parameters are only related to the two universal confluent exponents. However, the numerical values of $\Delta$ appear also conditioned by the errorbar propagation of asymptotic uncertainties provided by the theoretical estimations of the leading universal exponents close to the non-Gaussian fixed point. In Figure 3 we have reported by crosses at constant $\frac{\Delta+\Delta_{m f}}{2}$ as a function of $t^{*}$, all the [max, min, and mixing] conditions $S_{P, 2}^{ \pm} \sqrt{t^{*}}=1$, whatever $P$ and \pm states (extending then the previous results reported in Figure 2 for the homogeneous susceptibility case).

Since the dispersion of the mixing conditions $\sqrt{S_{P, \max , 2}^{ \pm} S_{P, \min , 2}^{ \pm} t^{*}}=1$ is significatively lowered, Figure 3 supports the possibility of choosing a unique value for $S_{2}$.

Moreover, anticipating the $S_{2}$ estimation given in the following subsection, the resulting mean value $S_{2}=$ 22.9007, common to all the properties and all the states, provides a condition $S_{2} \sqrt{t_{0}^{*}}=1$ in close agreement with the previous mixed conditions, as shown by the corresponding vertical line in Figure 3] The departure of the non-homogenous heat capacity case is probably due to the cumulative effect of the error bar propagation of the uncertainties on the exponent and on the critical background amplitude estimations, related here to the 


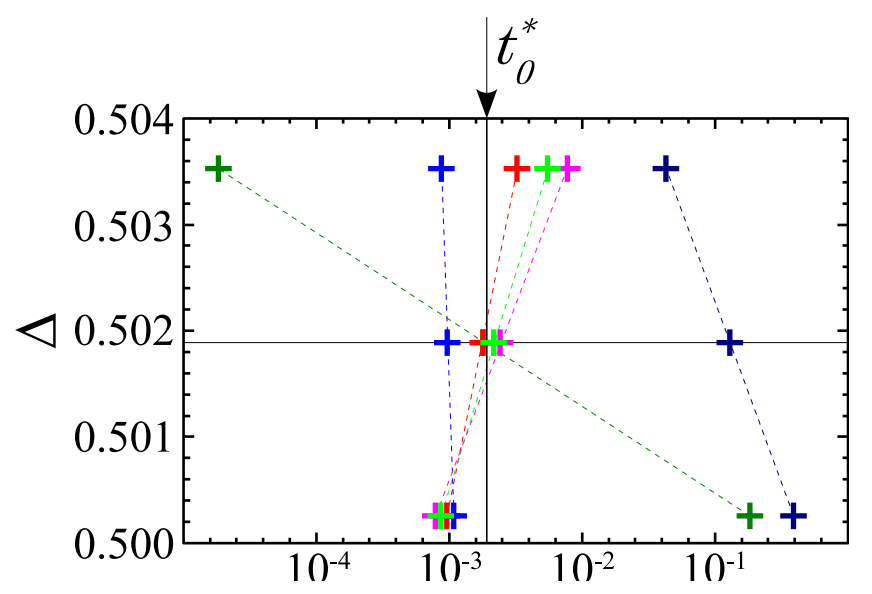

Figure 3: Characteristic values of the crossover condition $S_{P, 2}^{ \pm}\left(\Delta, \Delta_{m f}\right) \sqrt{t^{*}}=1$, for all the "min" (upper crosses at same $\Delta_{\max }$ value) and "max" (lower crosses at same $\Delta_{\min }$ value) crossover functions estimated in Ref. 14] (lin-log $\Delta ; t^{*}$ diagram). The $P$ (property) color indexations are: green $\chi^{*}(+)$, red $\ell^{*}(+)$, blue $C^{*}(+)$, pink $m^{*}(-)$, dark green $\chi^{*}(-)$, and dark blue $C^{*}(-)$. The corresponding values for the mixing crossover functions with $E=\frac{1}{2}$ [see Eq. (29)], are indicated by the intermediate crosses (at same $\Delta_{\text {mean }}=\frac{\Delta_{\max }+\Delta_{\min }}{2}$ value). The linear segments show the agreement with the associated geometrical mean values $\sqrt{S_{P, \max , 2}^{ \pm} S_{P, \min , 2}^{ \pm} t^{*}}=1$ of the min and max crossover conditions. The vertical line illustrates the "universal" crossover condition $S_{2} \sqrt{t_{0}^{*}}=1$ [where $D\left(\frac{1}{\left[S_{2}\left(\Delta_{\text {mean }}, \Delta_{m f}\right)\right]^{2}}\right)=$ $\frac{\Delta_{\text {mean }}+\Delta_{m f}}{2}$ from Eq. (6)], which is selected in the present study, whatever the property $(P)$ or the phase domain $( \pm)$.

non-zero value of the regular background term (equal to 3 ) below $T_{c}$. We can then note that the above wellcontrolled origin of this "universal" model-parameter $S_{2}$, partly compensates for the arbitrariness of its numerical value.

\subsection{Unique form of the mean confluent function}

For each property, the three associated functions,

$$
F_{P, t h}\left[t^{*}, D_{P}^{ \pm}\left(t^{*}\right)\right]=\left\{\begin{array}{c}
F_{P, \max }\left[t^{*}, D_{P, \text { max }}^{ \pm}\left(t^{*}\right)\right] \\
F_{P, \text { min }}\left[t^{*}, D_{P, \text { max }}^{ \pm}\left(t^{*}\right)\right] \\
F_{P, \text { mix }}^{E=\frac{1}{2}}\left[t^{*}, D_{P, \text { mix }}^{ \pm}\left(t^{*}\right)\right]
\end{array}\right\},
$$

proposed in reference [14 can be considered. The three corresponding residual functions, $r_{P}\left(t^{*}\right)$, expressed in $\%$, are calculated from reference to our mean function noted $F_{P}\left[t^{*}, D\left(t^{*}\right)\right]$, such as $r_{P}\left(t^{*}\right)=100 \times$ $\left(\frac{F_{P, t h}\left[t^{*}, D_{P}^{ \pm}\left(t^{*}\right)\right]}{F_{P}\left[t^{*}, D\left(t^{*}\right)\right]}-1\right)$. The results reported hereafter are obtained from the minimization method of the residuals

\begin{tabular}{|c|c|c|c|c|}
\hline 0 & $P$ & $1 / \ell^{*}$ & $1 / \chi^{*}$ & $C^{*}$ \\
\hline 1 & $e_{P}^{+}$ & -0.6303875 & -1.2395935 & 0.1088375 \\
\hline 2 & $\mathbb{Z}_{P}^{+}$ & 2.121008 & 3.709601 & 1.719788 \\
\hline 3 & $\Delta$ & 0.50189 & & \\
\hline 4 & $\mathbb{Z}_{P}^{1,+}$ & -5.81623 & -8.56347 & 8.06569 \\
\hline 5 & $S_{1}$ & 22.8573 & & \\
\hline 6 & $S_{2}$ & 22.9007 & & \\
\hline 7 & $X_{P, 1}$ & 40.0606 & 29.1778 & 36.6874 \\
\hline 8 & $Y_{P, 1}$ & -0.098968 & -0.178403 & 0.220033 \\
\hline 9 & $X_{P, 2}$ & 11.93211 & 11.7625 & 3.23787 \\
\hline 10 & $Y_{P, 2}$ & -0.153912 & -0.282241 & -0.000133095 \\
\hline 11 & $X_{P, 3}$ & 1.902735 & 2.05948 & 2.84102 \\
\hline 12 & $Y_{P, 3}$ & -0.00789505 & -0.0185424 & -0.00222489 \\
\hline 13 & $X_{C}$ & & & -3.79829 \\
\hline 14 & $e_{P, m f}$ & -0.5 & -1 & 0 \\
\hline 15 & $\mathbb{Z}_{P, m f}^{+}$ & 1 & 1 & 3.79004 \\
\hline 16 & $\Delta_{m f}$ & -0.5 & & \\
\hline 17 & $\mathbb{Z}_{P, m f}^{1,+}$ & -0.0195196 & -0.0391128 & 0.00517327 \\
\hline
\end{tabular}

Table I: Numerical values of the parameters of the mean crossover functions $F_{P}\left[t^{*}, D\left(t^{*}\right)\right]$ [see Eqs. (5) and (6), with $K=3$ and $S_{P, 2}^{ \pm}=S_{2}$, corresponding to the dimensionless correlation length $\ell^{*}$, susceptibility $\chi^{*}$, and specific heat $C^{*}$, in the homogeneous phase $\left(T>T_{c}\right)$. The lines 1 to 4 correspond to the characteristic parameters of the Ising-like PAD description [see Eqs. (24) to (28)], while the lines 14 to 17 correspond to the characteristic parameters of the mean fieldlike PAD description [see Eqs. (32) to 37. The universal values of the interrelated crossover sensors $S_{1}$ and $S_{2}$ [see Eqs. [23)], are given in the lines 5 and 6 . The values of the three amplitude-exponent pairs $\left\{X_{i}, Y_{i}\right\}_{P}^{+}$given in lines 7 to 12 , have been determined by a carefull adjustement to the theoretical mixing functions of Eq. (29) (with $E=\frac{1}{2}$ ) proposed in Ref. 14], using specific constraints of Equations (25) to (28) and (34) to (37). The value of the critical background $X_{C}$ of the specific heat given in line 13 is the mean value of the min and max values of parameters $X_{6}$ of Ref. 14].

related only to the mixing function with $E=\frac{1}{2}$ [see Eq. 29 .

In a first step, starting with the susceptibility in the homogeneous phase as a basic property (since $K=3$ already), we have validated the derivation of several mean functions using several entry triads made of $\sqrt{S_{\chi, \max , 2}^{+} S_{\chi, \min , 2}^{+}}$and one among the three pairs $\left\{X_{i, \max }, Y_{i, \max }\right\}_{\chi}^{+}, \quad\left\{X_{i, \min }, Y_{i, \min }\right\}_{\chi}^{+}$, or $\left\{\sqrt{X_{i, \max } X_{i, \min }}, \frac{Y_{i, \max }+Y_{i, \min }}{2}\right\}_{\chi}^{ \pm}$for each $i$ value between 1 to $K$. The two remaining pairs $\left\{X_{j \neq i}, Y_{j \neq i}\right\}_{\chi}^{+} \mathrm{s}$ were then calculated from Eqs.(28), (34), (35) and (37). This step was then repeated for all the properties with two main results:

1. at least one solution with $K=3$ exists for all the properties and all the states; 


\begin{tabular}{|c|c|c|c|c|c|}
\hline 0 & $P$ & $m^{*}(\mathrm{MR} 7)$ & $m^{*}(\mathrm{MR} 67)$ & $1 / \chi^{*}$ & $C^{*}$ \\
\hline 1 & $e_{P}^{-}$ & 0.3257845 & 0.3257845 & -1.2395935 & 0.1088375 \\
\hline 2 & $\mathbb{Z}_{P}^{-}$ & 0.937528 & 0.937528 & 17.762821 & 3.203771 \\
\hline 3 & $\Delta$ & 0.50189 & 0.50189 & & \\
\hline 4 & $\mathbb{Z}_{P}^{1,-}$ & 3.42538 & 7.70712 & -40.4666 & 6.69984 \\
\hline 5 & $S_{1}$ & 22.8573 & 22.8573 & & \\
\hline 6 & $S_{2}$ & 22.9007 & 22.9007 & & \\
\hline 7 & $X_{P, 1}$ & 219.597 & 124.274 & 470.671 & 116.515 \\
\hline 8 & $Y_{P, 1}$ & -0.0284374 & 0.0206744 & -0.0332665 & -0.105953 \\
\hline 9 & $X_{P, 2}$ & 38.3772 & 20.9461 & 43.6468 & 72.9532 \\
\hline 10 & $Y_{P, 2}$ & 0.224464 & 0.20453 & -0.585082 & 0.247902 \\
\hline 11 & $X_{P, 3}$ & 6.92804 & 6.92808 & 5.2264 & 12.6739 \\
\hline 12 & $Y_{P, 3}$ & 0.152404 & 0.123226 & 0.139161 & 0.0757262 \\
\hline 13 & $X_{C}$ & & & & -3.79349 \\
\hline 14 & $e_{P, m f}$ & 0.5 & 0.5 & -1 & 0 \\
\hline 15 & $\mathbb{Z}_{P, m f}^{-}$ & $\sqrt{6}$ & $\sqrt{6}$ & 2 & 6.79349 \\
\hline 16 & $\Delta_{m f}$ & -0.5 & -0.5 & & \\
\hline 17 & $\mathbb{Z}_{P, m f}^{1,-}$ & 0.0277176 & 0.0277175 & 0.0131516 & 0.00846373 \\
\hline
\end{tabular}

Table II: Same as Table I for the dimensionless order parameter $m^{*}$, susceptibility $\chi^{*}$, and specific heat $C^{*}$, in the non-homogeneous phase $\left(T<T_{c}\right)$. The distinction between the MR7 and MR 67 results accounts for difference in the numerical values of the first confluent amplitude $\mathbb{Z}_{M}^{1}$ of Eq. (28), which provides the (central) universal values $\frac{\mathbb{Z}_{M}^{1}}{\mathbb{Z}_{\chi}^{1++}}(M R 7)=0.45$ and $\frac{\mathbb{Z}_{M}^{1}}{\mathbb{Z}_{\chi}^{1,+}}(M R 67) \equiv \frac{\mathbb{Z}_{M}^{1}}{\mathbb{Z}_{\chi}^{1++}}(M R 6)=0.9$ (see Appendix B for details).

2. the residuals are minimum for the triad $\left\{\sqrt{S_{\max , 2} S_{\min , 2}}, \sqrt{X_{i, \max } X_{i, \min }}, \frac{Y_{i, \max }+Y_{i, \min }}{2}\right\}_{P}^{ \pm}$.

The following complementary observations were also made:

1. The significative values of residuals are in a $t^{*}$-range where $S_{P, 2}^{ \pm} \sqrt{t^{*}} \simeq 1$.

2. The $t^{*}$-range where our mean functions compare to the $\max$ (or min) functions, corresponds to $t^{*}>$ $\frac{0.1}{S_{P, 2}^{ \pm}}$(in this domain, the residuals are of the same order of magnitude than those obtained using the $\left(E=\frac{1}{2}\right)$ mixing functions and remains $\left.\lesssim 10^{-3}\right)$.

3. Each Ising-like PAD description by equation (24) agrees with the complete crossover function within an error-bar lower than $0.01 \%$ for $t^{*} \leq \mathcal{L}_{P A D}^{I s i n g}$, where $\mathcal{L}_{P A D}^{I \operatorname{sing}}$ is then defined by the value

$$
\left(S_{P, 2}^{ \pm}\right)^{2} \mathcal{L}_{P A D}^{I \operatorname{sing}} \simeq 10^{-3}
$$

In a second step, for the inverse susceptibility and the inverse correlation length in the homogeneous state, we have minimized the residuals by successive small variations around the above mixing values of each constitutive parameter of the entry triad. We have then selected the best mean functions for each property, using the mean value $S_{2}=22.9007$ of the two optimized values $S_{\chi, 2}=22.9321$ and $S_{\xi, 2}=22.8693$, associated to $\chi$ and $\xi$, respectively. The final step was to minimize all the residuals with $S_{2}=22.9007$ fixed, whatever the property or the domain. As a main consequence, the universal confluent crossover condition $S_{2} \sqrt{t^{*}}=1$ is such that $t^{*}=t_{0}^{*}$, with

$$
t_{0}^{*}=\frac{1}{\left(S_{2}\right)^{2}} \cong 2 \cdot 10^{-3}
$$

Accordingly, Eq. (38) has a universal form whatever the property, leading to well-controlled PAD extension $t^{*} \leq$ $\mathcal{L}_{P A D}^{I s i n g}$, with

$$
\mathcal{L}_{P A D}^{I \text { sing }} \simeq \frac{10^{-3}}{\left(S_{2}\right)^{2}}=\varpi \times t_{0}^{*} \cong 2.10^{-6}
$$

where $\varpi \cong 10^{-3}$ is then a convenient parameter to account for the intermediate crossover range between the two (Ising like and mean field like) PADs (see Figure 2 for the susceptibility case as a typical example ).

The corresponding parameters of the theoretical mean functions are given in Table凹(homogeneous state, $\Delta \tau^{*}>$ 0 ) and Table (non-homogeneous state, $\left.\Delta \tau^{*}<0\right)$. The mean crossover functions for the susceptibility, the correlation length and specific heat in the homogeneous domain are within the above theoretical level of precision in the complete $t^{*}$-range. For the order parameter, susceptibility and specific heat in the non-homogeneous domain we get a sufficient accuracy $( \pm 0.1 \%)$ to have agreement with the theoretical crossover shape in the intermediate $t^{*}$-range. The MR67 order parameter case in the nonhomogeneous domain is the object of a specific analysis (see Appendix B) related to the application restricted to the one component fluid subclass. Correspondingly, for a more detailed analysis on the level of precision, we have also reported in this Appendix B the residuals for all the properties (see Figures [5] to [10).

To evaluate the influence of the constant universal value $S_{2}=$ cte $=22.9007$, we have made a comparison between all the $t_{e_{P}}^{*}$-values where the following local mean value

$$
e_{P, e f f}=e_{P, \frac{1}{2}}=\frac{e_{P}+e_{m f}}{2}
$$

of the effective exponent $e_{P, \text { eff }}$ occurs for any max, min, and mixing crossover function [the effective exponent is given by the equation $\left.e_{P, e f f}=-\frac{\partial \ln \left[F_{P}\left[t^{*}, D_{P}^{ \pm}\left(t^{*}\right)\right]\right]}{\partial \ln t^{*}}[32]\right]$.

In Figure 4 using a vertical arbitrary unit (a.u.) scale between $e_{P \min , \frac{1}{2}}(a . u)=$.0 to $e_{P \max , \frac{1}{2}}(a . u$. $)=1$, then with $e_{P, \frac{1}{2}}($ a.u. $)=e_{P \operatorname{mix}, \frac{1}{2}}($ a.u. $)=\frac{1}{2}$ at $t^{*}=t_{e_{P}}^{*}$, permits to generalize the (confluent) $\Delta$ case reported in Figure 3. We can observe that the conditions of Eq. (41) match perfectly at $t^{*}=t_{e_{P}}^{*}$ for mean crossover functions and mixing crossover functions.

Figure 4 is the second result supporting our $S_{2}=$ cte suggestion to construct the "universal" confluent function. The corresponding condition $\Delta_{\frac{1}{2}}=\frac{\Delta+\Delta_{m f}}{2}$ for 


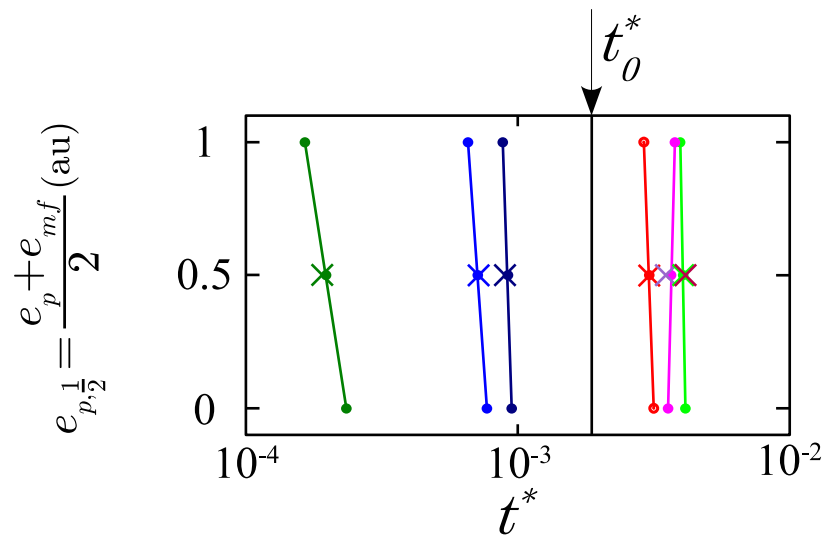

Figure 4: $t_{e_{P}}^{*}$-position (semi-log scale) of the effective mean value $e_{P, \frac{1}{2}}=\frac{e_{P}+e_{m f}}{2}$, (in arbitrary units), between critical and classical asymptotic exponents. Upper, lower, and median circles are for $e_{P} \max , \min$, and mixing values of reference [14], while median crosses are for mean values of the present mean crossover functions. See the legend of Figure 3 for the $P$ color indexation. The vertical (black) line indicates the $t_{0}^{*}$-position for the universal confluent condition $S_{2} \sqrt{t_{0}^{*}}=1$. Note the significative differences in $t_{e_{P}}^{*}$-positions for max, min, and mean values of the effective leading exponents for each property, compared to the "universal" effective confluent exponent case reported in Figure 3

the effective confluent exponent, where $S_{2} \sqrt{t_{0}^{*}}=1$ [Eq. [39] ], demonstrates that the $t_{e_{P}}^{*}$-values are readily "C3" in nature (see Figure 31), since, either the conditions $\frac{1}{10}\left(\frac{1}{S_{2}}\right)^{2} \lesssim t_{e_{P}}^{*} \lesssim 2\left(\frac{1}{S_{2}}\right)^{2}$, or the conditions $\left|\Delta_{\text {eff }}\left(t_{e_{P}}^{*}\right)-\Delta_{\frac{1}{2}}\right|<\frac{1}{2}\left|\Delta_{\text {Asymp }}-\Delta_{\frac{1}{2}}\right|$, with $\Delta_{\text {Asymp }}=$ $\Delta$ or $\Delta_{m f}$, are satisfied. We note that the relative $t^{*}$ differences between the values of $t_{\gamma}^{*}(-) \approx \frac{1}{10}\left(\frac{1}{S_{2}}\right)^{2}$, and $t_{\gamma}^{*}(+) \approx 2\left(\frac{1}{S_{2}}\right)^{2}$, for the inverse susceptibility in the non-homogeneous $(-)$, and homogeneous $(+)$, phases, is correctly reproduced, while the $t^{*}$-similarity, $t_{\alpha}^{*}(-) \approx$ $t_{\alpha}^{*}(+) \approx \frac{1}{3}\left(\frac{1}{S_{2}}\right)^{2}$ of the corresponding values for the specific heat, is also recovered (compare with the Figure 1 of Ref. [14]).

\section{CONCLUSION}

By construction, the mean theoretical crossover functions determined in the present work do not account for any uncertainty on the parameters which, in the preasymptotic critical domain (PAD), characterize the critical behavior of the functions considered (correlation length, susceptibility, specific heat and coexistence curve). The recourse to such mean functions is justified by the fact that the amplitude and propagation of the asymptotic error-bars have a large extension which can affect significantly the classical-to-critical crossover espe- cially in the intermediate region where most experimental data are reported. Actually, it is known that in such a situation, when the comparison with experimental data is made following the common way of trying to determine the leading and first confluent amplitudes in the Isinglike PAD, the theoretical and experimental uncertainties add to each other. Consequently, it is usual to fix the exponents to their mean theoretical values in order to get some definite information on the correction amplitudes. If we use the min and max theoretical crossover functions very close to the critical point, we obtain non-satisfying information on the intermediate crossover because the physical parameters $\vartheta$ and $\psi$, introduced by the basic Eqs. (12) and (13) are not sufficiently well defined. The mean functions constructed with controlled constraints which correctly account for the universal features proper to the Ising-like PAD, provide useful criteria to define the extension of the Ising-like PAD. Fitting the experimental data with our mean crossover functions can then be a convenient method to validate any phenomenological approach by predicting the values of the two-scale factors which characterize the asymptotic singular behavior within the Ising-like PAD and finally to identify eventual subclass of universality. In a forthcoming paper 15], the importance of the mean crossover functions will be illustrated by providing a complete characterization of the one component fluid subclass, within an extended asymptotic range well beyond the Ising like PAD, recovering then the practical intermediate range which is usually described by more complex formulations [33, 34].

\section{Appendix A: THE ADJUSTABLE PARAMETERS}

To simplify our analysis, we select the threedimensional Ising-like $\Phi_{d=3}^{4}(n=1)$ model, with $g_{0} \sim$ $[\text { length }]^{-1}$.

Let us consider the theoretical crossover function of Eq. (5) for the (dimensionless) correlation length $\ell^{*}\left(t^{*}\right)$ given in Table \at $h^{*}=0$ as a typical example, writing then

$$
\ell^{*}=g_{0} \times \xi_{\text {exp }}
$$

and [see Eq. (12)],

$$
t^{*}=\vartheta \times \Delta \tau^{*}
$$

Now fitting the experimental correlation length $\xi_{\exp }\left(\Delta \tau^{*}\right)$, in the homogeneous phase $\Delta \tau^{*}>0$, gives access to the two adjustable parameters $g_{0}$ and $\vartheta$. Consequently, due to the asymptotic validity of Eq. (A2) close to the critical point, is known the asymptotic two-term Wegner expansion of the experimental singular behavior for the correlation length, which reads as follows

$$
\xi_{e x p}=\xi_{0}^{+}\left(\Delta \tau^{*}\right)^{-\nu}\left[1+a_{\xi}^{+}\left(\Delta \tau^{*}\right)^{\Delta}+\ldots\right]
$$



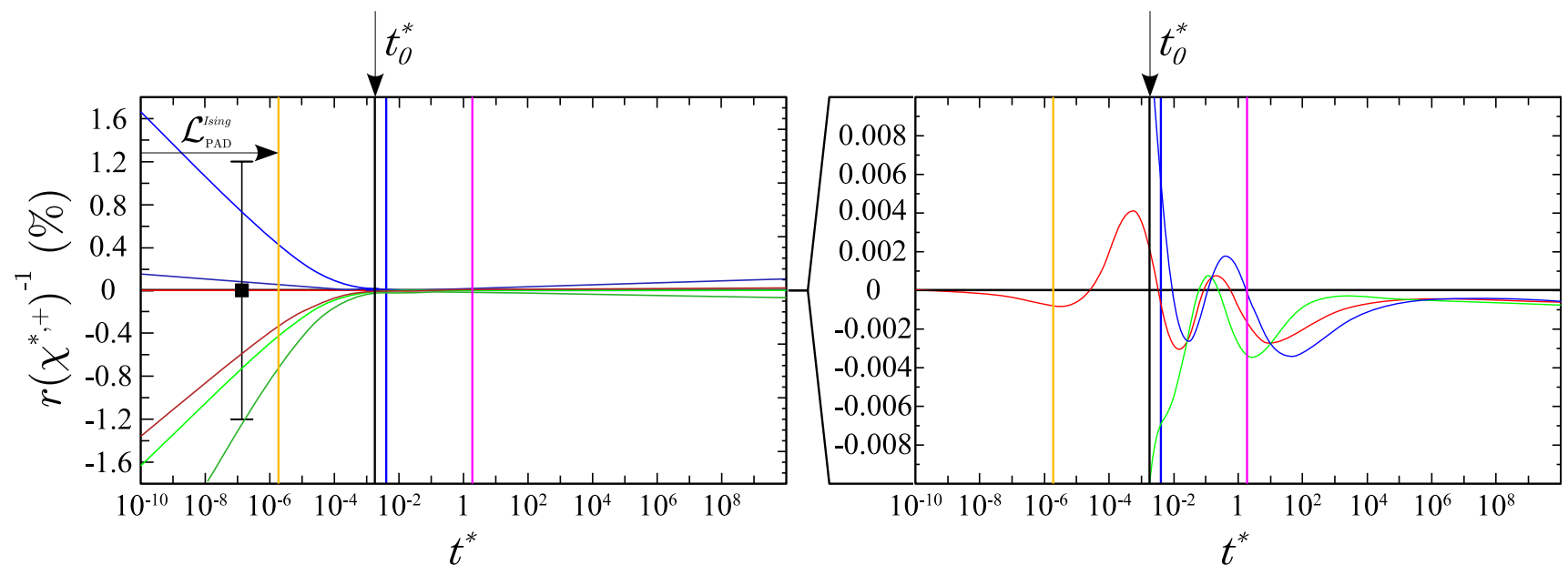

Figure 5: Residuals (\%) for the inverse susceptibility in the homogeneous phase. The right part compares relative residuals to the theoretical fitting precision of $0.01 \%$. Color indexation: green (dark green) : MR7 (MR6) min crossover function; blue (dark blue): MR7 (MR6) max crossover function; red (dark red): MR7 (MR6) mixing crossover function with $E=\frac{1}{2}$; Residuals for MR6 functions are not visible in the magnified rigth scale, except in the C3 range. Four characteristics $t^{*}$-positions are indicated by vertical lines which correspond to the $t^{*} \leq \mathcal{L}_{P A D}^{\text {Ising }}$ (i.e. the Ising like PAD extension) (orange line), the $S_{2} \sqrt{t_{0}^{*}}=1$ condition (black line), the $t^{*}=t_{\gamma}^{*}$ condition [Eq. [41)] (blue line), and $t^{*} \geq \mathcal{L}_{P A D}^{m f}$ (i.e. the mean field like PAD extension) (pink line), respectively (see text for details). The amplitude and propagation of the asymptotic theoretical error-bars for the non-gausssian limit $t^{*} \rightarrow 0$ are clearly evident in the left part. To compare the amplitude of the theoretical error-bar to the one of the experimental uncertainty, the squared point and its associated theoretical error-bar indicate "equivalence" between the model and the experimental situation encountered at the "lowest" temperature distance $T-T_{c}=1 \mathrm{mK}$ above the critical temperature of xenon (see Ref. [15] for details). The rigth part corresponds to the residual magnification at the scale of the allowed [14] theoretical precision level $(\leq 0.01 \%)$.
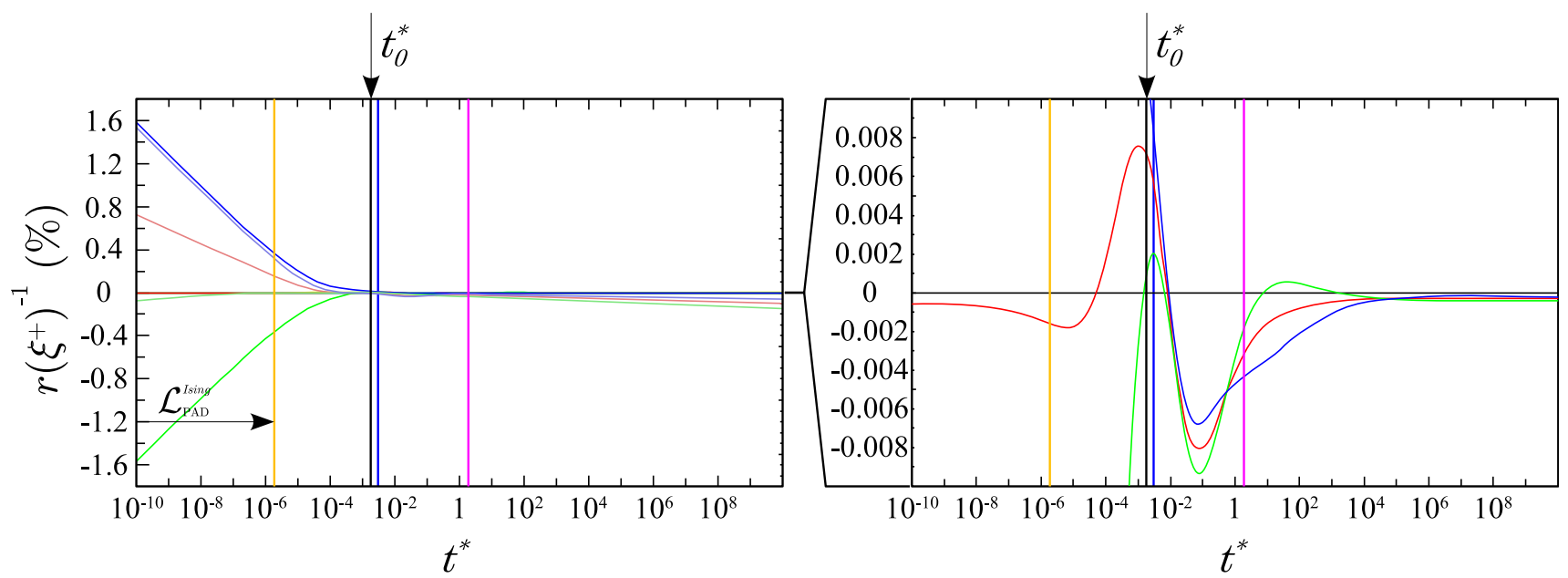

Figure 6: Same as Figure 4 for the inverse correlation length in the homogeneous phase.

The associated two-term expansion of the theoretical function reads as follows

$$
\ell^{*}=\left(\mathbb{Z}_{\ell}^{+}\right)^{-1}\left(t^{*}\right)^{-\nu}\left[1-\mathbb{Z}_{\ell}^{1,+}\left(t^{*}\right)^{\Delta}+\ldots\right]
$$

Using Eqs. A1A2, a term-to-term identification be- tween Eqs. A3 and (A4), gives,

$$
\vartheta=\left(\frac{a_{\xi}^{+}}{-\mathbb{Z}_{\ell}^{1,+}}\right)^{\frac{1}{\Delta}}
$$

and

$$
g_{0}^{-1}=\xi_{0}^{+} \mathbb{Z}_{\ell}^{+} \vartheta^{\nu}
$$

Within the preasymptotic domain, the two adjustable parameters of the model at $h^{*}=0$ are unequivocally 

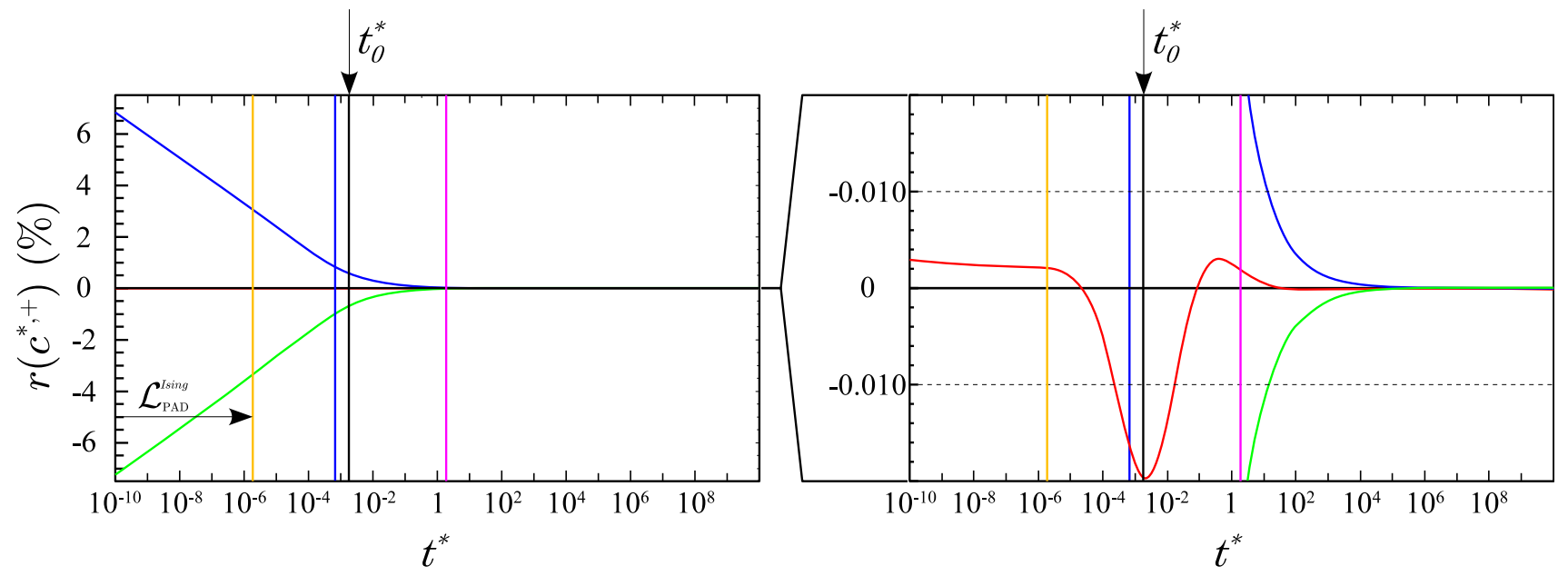

Figure 7: Same as Figure 4 for the specific heat in the homogenous phase. Compared to results of Figures 4 and 5 , the significative increases in amplitude and propagation of the error-bars is such as the residuals attain the admitted theoretical level in the $\mathrm{C} 3$ domain.
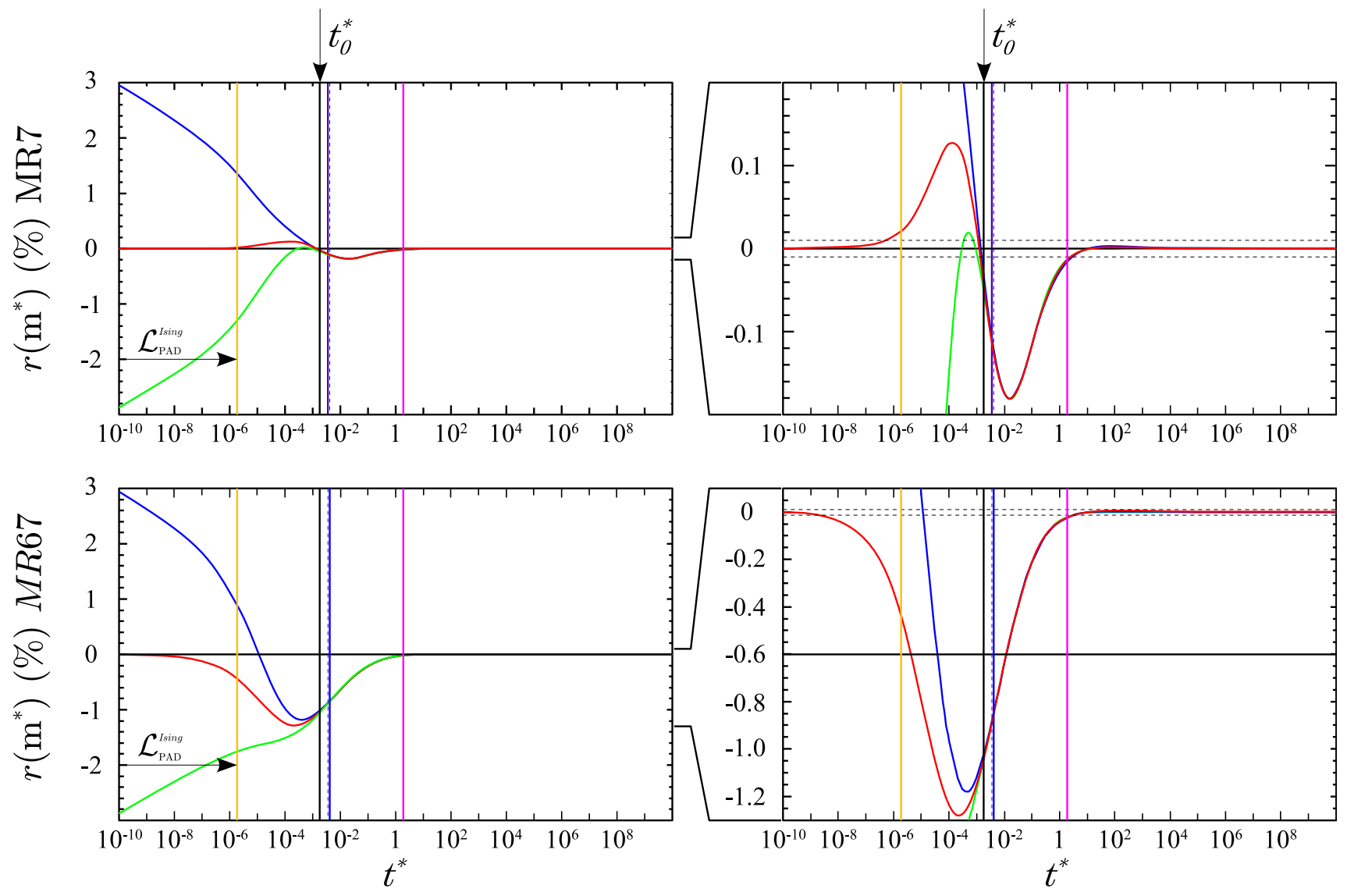

Figure 8: Residuals (\%) for the order parameter in the non-homogenous phase. See Appendix B for details between the MR7 (upper part) and MR 67 (lower part) results.

defined by Eqs. A5 and (A6). Two important remarks can be made:

i) the nonuniversal scale factor $\vartheta$ is uniquely defined by the confluent corrections to scaling; ii) the nonuniversal inverse length $g_{0}$ is proportional to the inverse of the leading amplitude of the actual correlation length.

$>$ From now on, the complete characterization of the 

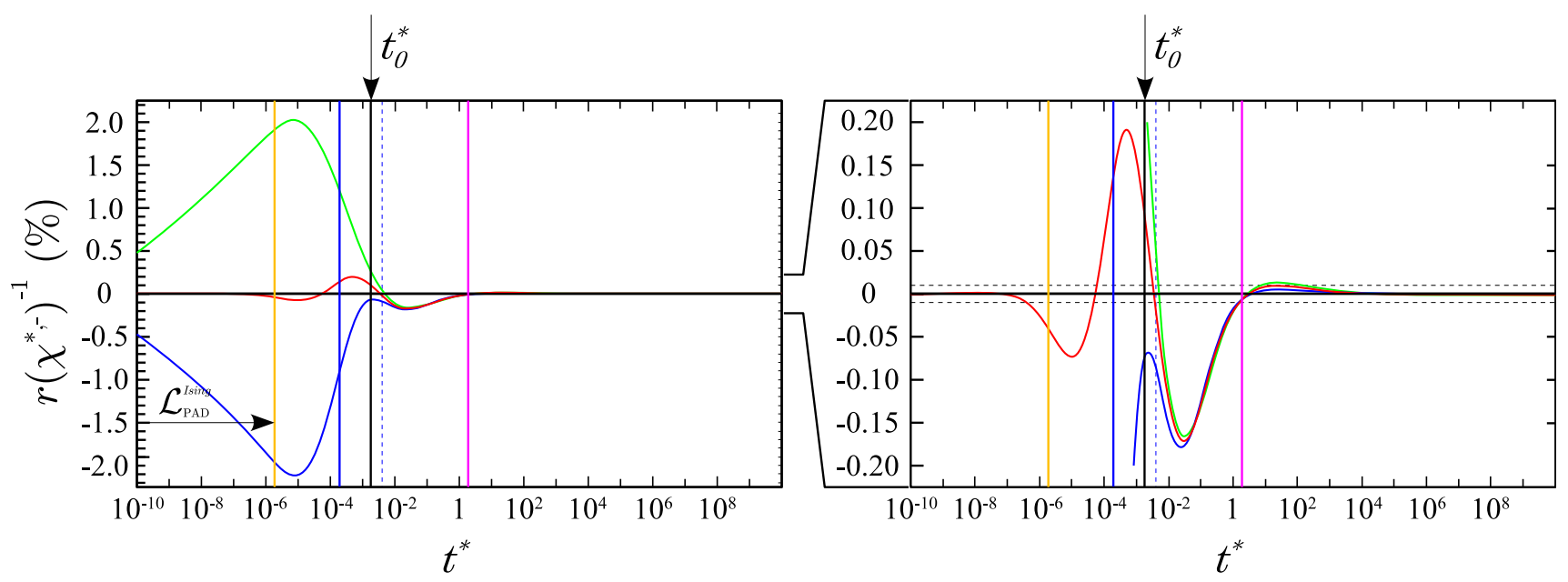

Figure 9: Same as Figure 4 for the susceptibility in the non-homogenous phase. Compared to the general trend of all the other max and min crossover functions, a curious "exact crossing" between min and max functions occurs in the present case around $t^{*} \simeq 310^{-13}$, as revealed by the converging residuals for $t^{*}<10^{-5}$. That provides the maximun amplitude for theoretical error-bars observed outside the PAD extension. The associated error-bar propagation is then significative in a large C3 domain.
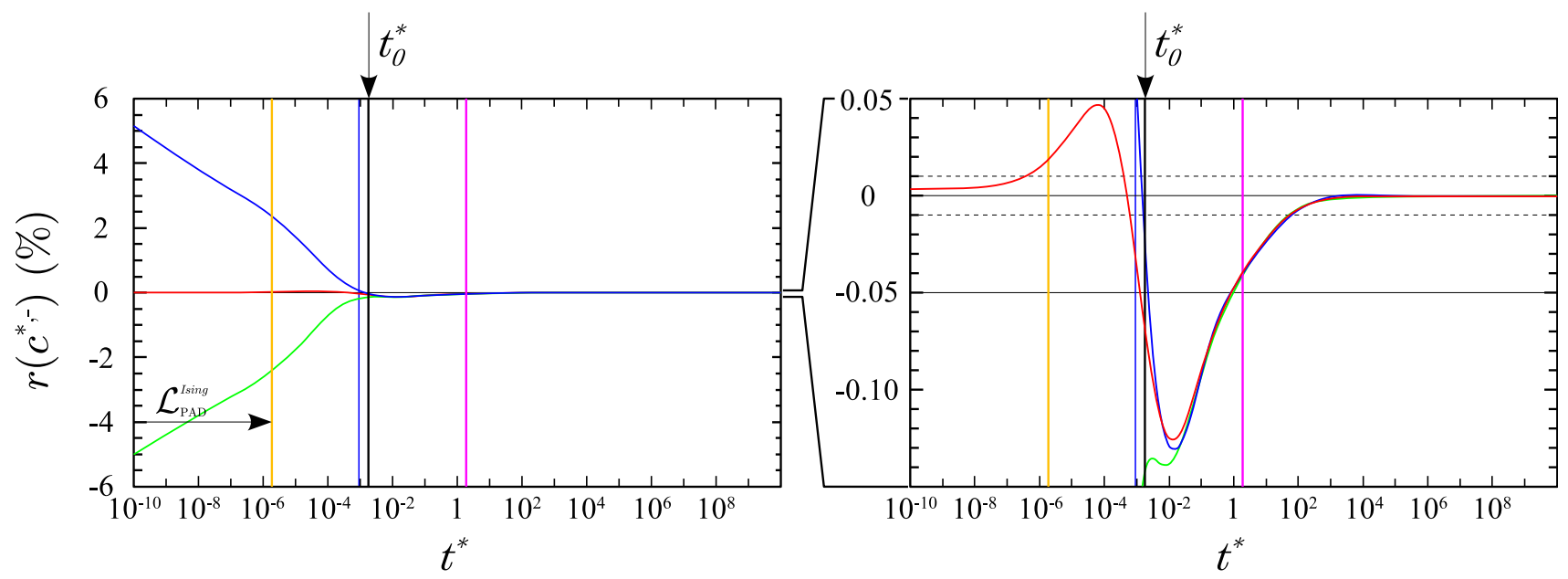

Figure 10: Same as Figure 4 for the specific heat in the non-homogenous phase. Compared to the homogeneous phase (Figure 6 ), we suspect that the non-zero value of the backgroung term contributes to increase amplitude and error-bar propagation over the C3 domain, leading to its over-extended range close to the Gaussian limit $t^{*} \rightarrow \infty$.

system would follow from the determination of $\psi$ which requires the consideration of one suplementary singular property, such as the susceptibility (as shown in the second paper [15]).

However, the inverse length $g_{0}$, determined from correlation length measurements, is not a "natural" scale for the actual system because a measurement of the correlation length would be a prerequisite condition to set the length scale unit. One may easily show 35] that when the thermodynamic description of a magnetic system is normalized per particle, then all lengths are measured in units of the thermodynamic microscopic length $a_{\text {mag }}=\left(v_{\text {mag }}\right)^{1 / d}$ (where $v_{\text {mag }}$ is the particle volume at criticality). In this respect, $a_{m a g}$ plays a role similar to that of the lattice spacing $a_{\text {Ising }}$ of the Ising uncompress- ible solid. The natural approach is thus to first choose a microscopic length unit such as $a_{\text {Ising }}$, which implicitly introduces the number of particles per lattice cell. Therefore, the value of $g_{0}$ is obtained via the length $a_{\text {Ising }}$ from the determination of an intermediate parameter $u_{I s i n g}^{*}$ [like in Eq. (15)] such as:

$$
g_{0} \times a_{\text {Ising }}=u_{I \operatorname{sing}}^{*}
$$

As a conclusion, fitting experimental results with the dimensionless theoretical functions requires reference to one length scale unit $\left[a_{\text {Ising }}\right.$, or $\left.a_{m a g}\right]$ and the energy scale unit $k_{B} T_{c}$ to reduce to dimensionless quantities the thermodynamic and correlation functions. The fitting results of correlation length and susceptibility measurements for example [15], enable the determination of 
three dimensionless numbers $\vartheta, \psi$, and $g_{0} \times a_{\text {Ising }}$ (or $\left.g_{0} \times a_{m a g}\right)$. In such a situation, $\vartheta$ and $\psi$ act as the two-scale factors characteristic of the asymptotic universal features of the Ising-like system, while the product $g_{0} \times a_{\text {Ising }}\left(\right.$ or $\left.g_{0} \times a_{\text {mag }}\right)$ insures that the extensivity of the total macroscopic system is correctly accounted for in units of the actual critical correlation length.

However, to compare between two systems of the same universality class remains not easy in the absence of explicit thermodynamic definition of the (coupling constant) inverse length $g_{0}$. This exercice is left to the second paper [15].

\section{Appendix B: THE COEXISTENCE CURVE AND ITS CONFLUENT CORRECTION ERROR-BAR}

As explained in Ref. [14], the MR7 crossover functions involve a forced account of the Guida and Zinn-Justin estimates [27] of the universal combinations between the leading critical amplitudes. This has induced an overestimation of the uncertainty on the correction terms in MR7 compared to the previous MR6 calculations (see section IIB2 of Ref. [14]). In general this does not have an important impact on the resulting mean crossover functions, except for the order parameter for which the MR7 determination finally appears to have (relatively to MR6) a poor quality (one may appreciate this difference by looking at bottom of Figures 2 (MR6) and 3 (MR7) of Ref. [22], for example).
To circumvent this non-satisfactory error-bar situation, we have applied the procedure described in Section 3 to construct a "modified" crossover function (labelled MR67) of the order parameter. Actually, our MR67 function incorporates the same mean value of the leading amplitude as the MR7 one, $\mathbb{Z}_{M}(M R 67)=$ $\mathbb{Z}_{M}(M R 7)=0.937528$, and combines the MR6 central value of the universal ratio $\frac{\mathbb{Z}_{M}^{1}}{\mathbb{Z}_{\chi}^{1,+}}(M R 6)=0.9$, with the MR7 amplitude value of $\mathbb{Z}_{\chi}^{1,+}(M R 7)=8.5635$, so that $\mathbb{Z}_{M}^{1}(M R 67)=7.70712$. Using such a practice, we have reported the difficulty of accounting for error bars in the MR7 calculations of the crossover functions on only one property in the non homogeneous range. In the forthcoming paper dedicated to the study of the one component fluid subclass [15], a detailed analysis of this particular choice will be presented.

The corresponding numerical values of the parameters of Eq. (5), needed to calculate the MR67 mean crossover function for the order parameter in non-homogeneous state $\left(t^{*}<0\right)$, are given in Table II

For a reader interested in checking the level of precision for the present mean crossover functions in the three Ising-like, intermediate, and mean-field like, $t^{*}$-range, we have reported the residuals $100 \times\left(\frac{F_{P, \max , \min , \operatorname{mix}}\left[t^{*}\right]}{F_{P, \text { mean }}\left(t^{*}\right)}-1\right)$ for all the properties (see Figures [5 to [10). The right part of each figures gives the appropriate magnified scale of the residuals to compare with the estimated precison $( \pm 0.01 \%)$ for the fitting theoretical functions.
[1] see for example J. Zinn-Justin, Euclidean Field Theory and Critical Phenomena, $4^{\text {th }}$ ed. (Clarendon Press, Oxford, 2002).

[2] K. G. Wilson and M. E. Fisher, Phys. Rev. Lett. 28, 240 (1972).

[3] K. G. Wilson and J. Kogut, Phys. Rep. 12C, 77 (1974).

[4] C. Bagnuls and C. Bervillier, J. Phys. (Paris) Lett. 45, L-95 (1984).

[5] C. Bagnuls and C. Bervillier, Phys. Rev. B 32, 7209 (1985).

[6] C. Bagnuls, C. Bervillier, D. I. Meiron and B. G. Nickel, Phys. Rev. B 35, 3585 (1987); Phys. Rev. B 65, 149901(E) (2002).

[7] R. Schloms and V. Dohm, Nucl. Phys. B 328, 639 (1989).

[8] R. Schloms and V. Dohm, Phys. Rev. B 42, 6142 (1990).

[9] Y. Garrabos, Thesis, University of Paris VI, 1982 (unpublished).

[10] Y. Garrabos, J. Phys. 46, 281 (1985); (for an english version see: e-print/cond-mat/0512347).

[11] Y. Garrabos, J. Phys. 47, 197 (1986).

[12] Y. Garrabos, B. Le Neindre, R. Wunenburger, C. Lecoutre-Chabot, and D. Beysens, Int. J. Thermophys. 23, 997 (2002).

[13] Y. Garrabos, F. Palencia, C. Lecoutre, C. J. Erkey, and B. Le Neindre, Phys. Rev. E 73, 021106 (2006).

[14] C. Bagnuls and C. Bervillier, Phys. Rev. E 65, 066132 (2002).
[15] Y. Garrabos, C. Lecoutre, F. Palencia, B. Le Neindre, and C. J. Erkey, preprint (2006).

[16] F. J. Wegner, Phys Rev. B 5, 4529 (1972).

[17] For a review of the definition of the field variables used in real fluids, see for example, M. A. Anisimov and J. V. Sengers, in Equations of State for Fluids and Fluid Mixtures, Part I, J.V. Sengers, R.F. Kayser, C.J. Peters, and H.J. White, Jr., Eds. (Elsevier, Amsterdam, UK, 2000) pp. 381-434, and references therein.

[18] For a complete account for mixing of the field variables, see for example, Y. C. Kim, M. E. Fisher, and G. Orkoulas, Phys. Rev. E 67, 061506 (2003), and references therein.

[19] C. Bagnuls, C. Bervillier, and Y. Garrabos, J. Phys. (Paris) Lett. 45, L-127 (1984).

[20] H. Güttinger and D. S. Cannell, Phys. Rev. A 24, 3188 (1981).

[21] C. Edwards, J. A. Lipa, and M. J. Buckingham, Phys. Rev. Lett. 20, 496 (1968); Phys. Rev. A 15, 778 (1977).

[22] F. Zhong and M. Barmatz, Phys. Rev. E 70, 066105 (2004).

[23] F. Zhong, M. Barmatz, and I Hahn, Phys. Rev. E 67, 021106 (2003).

[24] B. G. Nickel, D. I. Meiron and G. A. Baker, Jr, "Compilation of 2-pt and 4-pt graphs for continuous spin models", Guelph University preprint, unpublished (1977).

[25] D. B. Murray and B. G. Nickel, "Revised estimates for 
critical exponents for the continuum $\mathrm{n}$-vector model in 3 dimensions", unpublished (1991).

[26] J. C. Le Guillou and J. Zinn-Justin, Phys. Rev. B 21, 3976 (1980).

[27] R. Guida and J. Zinn-Justin, J. Phys. A: Math. Gen. 31, 8103 (1998).

[28] R. Guida and J. Zinn-Justin, Nucl. Phys. B 489, 626 (1997).

[29] C. Bagnuls and C. Bervillier, Phys. Rep. 348, 91 (2001).

[30] C. Bagnuls and C. Bervillier, Phys. Lett. A 195, 16 (1994).

[31] C. Bagnuls and C. Bervillier, Cond. Matt. Phys. 3, 559
(2000).

[32] J. S. Kouvel and M. E. Fisher, Phys. Rev. 136, A 1626 (1964).

[33] V. A. Agayan, M. A. Anisimov, and J. V. Sengers, Phys. Rev. E 64, 026125 (2001).

[34] S. B. Kiselev and J. F. Ely, J. Chem Phys. 119, 8645 (2003).

[35] V. Privman, P. C. Hohenberg, and A. Aharony, in "Phase Transitions and Critical Phenomena", Vol. 14, Ed. C. Domb and J. B. Lebowitz (Academic Press, New York, 1991). 